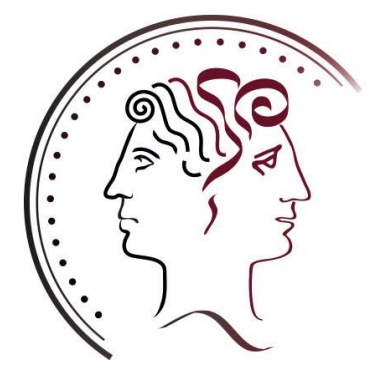

Working Papers of the Priority Programme 1859

Experience and Expectation.

Historical Foundations of Economic Behaviour

Edited by Alexander Nützenadel und Jochen Streb

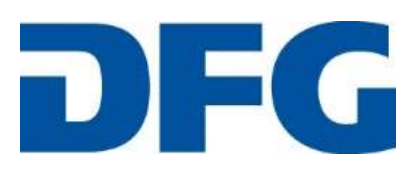

No 17 (2020, May)

Ettmeier, Stephanie / Kriwoluzky, Alexander

Active, or passive? Revisiting the role of

fiscal policy in the Great Inflation 
Arbeitspapiere des Schwerpunktprogramms 1859 der Deutschen Forschungsgemeinschaft „Erfahrung und Erwartung. Historische Grundlagen ökonomischen Handelns“ /

Working Papers of the German Research Foundation's Priority Programme 1859

"Experience and Expectation. Historical Foundations of Economic Behaviour"

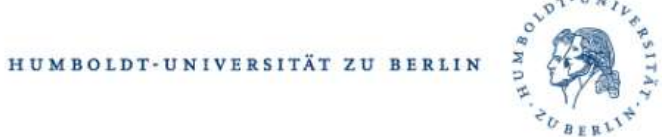

Published in co-operation with the documentation and publication service of the Humboldt University, Berlin (https://edoc.hu-berlin.de).

ISSN: 2510-053X

Redaktion: Alexander Nützenadel, Jochen Streb, Ingo Köhler

V.i.S.d.P.: Alexander Nützenadel, Jochen Streb

SPP 1859 "Erfahrung und Erwartung. Historische Grundlagen ökonomischen Handelns"

Sitz der Geschäftsführung:

Humboldt-Universität

Friedrichstr. 191-193, 10117 Berlin

Tel: 0049-30-2093-70615, Fax: 0049-30-2093-70644

Web: https://www.experience-expectation.de

Koordinatoren: Alexander Nützenadel, Jochen Streb

Assistent der Koordinatoren: Ingo Köhler

Recommended citation:

Ettmeier, Stephanie / Kriwoluzky, Alexander (2020): Active, or passive? Revisiting the role of fiscal policy in the Great Inflation, Working Papers of the Priority Programme 1859 "Experience and Expectation. Historical Foundations of Economic Behaviour" No 17 (May), Berlin

(C) 2020 DFG-Schwerpunktprogramm 1859 „Erfahrung und Erwartung. Historische Grundlagen ökonomischen Handelns"

The opinions and conclusions set forth in the Working Papers of the Priority Programme 1859 Experience and Expectation. Historical Foundations of Economic Behaviour are those of the authors. Reprints and any other use for publication that goes beyond the usual quotations and references in academic research and teaching require the explicit approval of the editors and must state the authors and original source. 


\title{
Active, or passive? Revisiting the role of fiscal policy in the Great Inflation*
}

\author{
Stephanie Ettmeier ${ }^{\dagger}$ Alexander Kriwoluzky
}

May 18, 2020

\begin{abstract}
We reexamine whether pre-Volcker U.S. fiscal policy was active or passive. To do so, we estimate a DSGE model with monetary and fiscal policy interactions employing a sequential Monte Carlo algorithm (SMC) for posterior evaluation. Unlike existing studies, we do not have to treat each policy regime as distinct, separately estimated, models. Rather, SMC enables us to estimate the DSGE model over its entire parameter space. A differentiated perspective results: pre-Volcker macroeconomic dynamics were similarly driven by a passive monetary/passive fiscal policy regime and fiscal dominance. Fiscal policy actions, especially government spending, were critical in the pre-Volcker inflation build-up.
\end{abstract}

JEL classification: C11, C15, E63, E65

Keywords: Bayesian Analysis, DSGE Models, Monetary-Fiscal Policy Interactions, Monte Carlo Methods

${ }^{*}$ We thank Khalid ElFayoumi, Sinem Hacioglu Hoke, Chi Hyun Kim, Martin Kliem, Eric Leeper, Gernot Müller, Frank Schorfheide, Moritz Schularick, and Nora Traum, as well as participants at the DIW Workshop on Fiscal Policy in Times of Crisis 2019, the RCEA Bayesian Econometrics Workshop 2019, the CEF 2019, the EEA-ESEM 2019, the Annual Meeting of the Verein für Socialpolitik 2019, and the Empirical Macroeconomics Seminar at Freie Universität Berlin. We also thank the HPC Service of ZEDAT, Freie Universität Berlin, for computing time.

${ }^{\dagger}$ DIW Berlin, and Freie Universität Berlin, Germany

${ }^{\ddagger}$ Corresponding author: DIW Berlin and Freie Universität Berlin, Germany, akriwoluzky@diw.de, +49 3089789430 


\section{Introduction}

With the inevitable COVID-19 fiscal responses, the global sovereign debt level is set to rise to a record high. The need to prevent situations in which debt becomes unsustainable puts surprise inflation on the table as a policy option to reduce nominal debt. The preceding financial crisis taught economists and policy makers alike that this is easier said than done. Since 2009, for instance, the European Central Bank tries very hard to reach its inflation target of close to two percent. Yet, euro-zone inflation is still lower. The looming recession and the expected lower global aggregate demand due to the pandemic will apply additional deflationary pressures. The fiscal theory of the price level (FTPL) suggests an alternative way: in a situation in which the fiscal authority is not commited to stabilize debt by managing the primary surplus and the monetary authority acts acommodatively, increasing government spending could be an option to fight deflationary pressure (Leeper et al., 2017). ${ }^{1}$

Interestingly, for one historical episode, which is very instructive for all these aspects, the debate about the monetary-fiscal policy mix is still unsettled. This episode is usually referred to as the Great Inflation of the 1960s and 1970s in the U.S. In our study, we revisit the role of fiscal policy for the Great Inflation to obtain insights for potential policy options in the current economic crisis. We estimate a DSGE model with three distinct monetary/fiscal policy regimes with a sequential Monte Carlo algorithm (SMC). Compared to previous studies, which estimated each possible policy regime as a distinct model, employing an SMC enables us to estimate the DSGE model over its entire parameter space. We find that the macroeconomic dynamics during the pre-Volcker period were almost similarly driven by a passive monetary/passive fiscal policy regime and fiscal dominance. Fiscal policy actions, in particular government spending, played an important role in the build-up of pre-Volcker inflation.

Meanwhile, the insight that monetary and fiscal policy are not independent from each

\footnotetext{
${ }^{1}$ Bianchi et al. (2020) propose a concrete policy that involves coordination between the monetary and fiscal authorities in response to the COVID-19 pandemic.
} 
other and must be studied jointly has a long tradition in modern macroeconomics, going back to Leeper (1991), Sims (1994), Woodford (1996), and Cochrane (2001). While an active policy authority is able to realize its agenda, the passive authority is reduced to accommodating the actions of counterparts. The literature largely agrees that monetary policy in the preVolcker period was passive. Clarida et al. (2000) and Mavroeidis (2010) estimate monetary policy reaction functions. Lubik and Schorfheide (2004) consider a monetary DSGE model that allows for indeterminacy, Boivin and Giannoni (2006) combine evidence from vector autoregressive and general equilibrium analysis, while Coibion and Gorodnichenko (2011), including the trend level of inflation in their study, arrive at a similar conclusion. However, concerning the stance of fiscal policy, the evidence is mixed. Bhattarai et al. (2016), who estimate a fixed-regime DSGE model with monetary and fiscal policy interactions, find that the fiscal authority was passive and strongly increased taxes to debt. On the contrary, studies relying on regime-switching DSGE models like Davig and Leeper (2006), Bianchi (2012), Bianchi and Ilut (2017), and Chen et al. (2019) mainly attribute the leading role in the pre-Volcker period to the fiscal authority.

To shed more light on this classic debate, we revisit the role of U.S. fiscal policy during the Great Inflation with a novel empirical toolkit. Specifically, the SMC algorithm, established in the DSGE literature by Herbst and Schorfheide (2014, 2015), allows us to create new perspectives on an old question. Previous studies relying on Bayesian-estimated fixed-regime DSGE models with distinct monetary/fiscal policy regimes, treated each regime as a different model. To determine the prevailing policy mix, typically each regime was successively imposed by estimating a restricted parameter space, draws from the corresponding posterior density were generated by a Markov chain Monte Carlo (MCMC) algorithm, usually the random walk Metropolis-Hastings algorithm (RWMH), and finally the fit of each model was compared by a model selection criterion. We argue that employing an SMC algorithm for drawing conclusions on the stance of monetary and fiscal policy is beneficial in three ways. First, the SMC is able to deal with difficult posterior surfaces, an outcome that a priori can- 
not be excluded in the case of a DSGE model with monetary-fiscal policy interactions. Due to this feature, the SMC enables us to estimate the DSGE model over its entire parameter space such that the most likely policy regime is directly determined by the data. Second, the SMC is amenable to parallelization and, thus computationally attractive, especially for larger DSGE models. Third, the SMC can be easily initialized by taking independent draws from the prior density. A time-consuming search for a mode is not necessary.

The remainder of this paper is as follows. Section 2 describes the DSGE model with monetary-fiscal policy interactions. In Section 3, we outline how we estimate the model. We iterate the status-quo in the empirical literature (RWMH posterior sampling) and explain why SMC sampling is the superior approach for models with multiple regimes. In Section 4, we discuss the estimation results to determine the monetary-fiscal policy mix in the preVolcker period. In the light of the estimation results, in Section 5 we examine what caused the build-up of U.S.-inflation in the 1960s and 1970s. The final section concludes the study.

\section{A DSGE model with monetary and fiscal policy in- teractions}

In this section, we outline the DSGE model with monetary-fiscal policy interactions, characterize its distinct monetary-fiscal policy regimes, and present the solution method for the model.

\subsection{Model description}

We use the DSGE model set up in Bhattarai et al. (2016). It features a complete description of fiscal policy, a time-varying inflation and debt-to-output target, partial dynamic price indexation, and external habit formation in consumption. Here, we only present the firstorder approximations of the model equations that determine equilibrium dynamics. For a detailed analysis of the model's characteristics, we refer the reader to the original study. 
Consumption behavior of households is given by the consumption Euler equation:

$$
\begin{array}{r}
\hat{C}_{t}=\frac{\bar{a}}{\bar{a}+\eta} E_{t} \hat{C}_{t+1}+\frac{\eta}{\bar{a}+\eta} \hat{C}_{t-1}-\left(\frac{\bar{a}-\eta}{\bar{a}+\eta}\right)\left(\hat{R}_{t}-E_{t} \hat{\pi}_{t+1}\right)+\frac{\bar{a}}{\bar{a}+\eta} E_{t} \hat{a}_{t+1}- \\
-\frac{\eta}{\bar{a}+\eta} \hat{a}_{t}+\left(\frac{\bar{a}-\eta}{\bar{a}+\eta}\right) \hat{d}_{t}
\end{array}
$$

where $\hat{C}_{t}$ is aggregate consumption, $\hat{R}_{t}$ is the interest rate on government bonds, $\hat{a}_{t}$ is the growth rate of technology, $\hat{\pi}_{t}$ is the inflation rate, and $\hat{d}_{t}$ stands for preferences. ${ }^{2}$ The parameters $\bar{a}$ and $\eta$ denote the steady-state value of $a_{t}$ and external habit formation, respectively.

The New Keynesian Phillips curve is denoted by

$$
\begin{array}{r}
\hat{\pi}_{t}=\frac{\beta}{1+\gamma \beta} E_{t} \hat{\pi}_{t+1}+\frac{\gamma}{1+\gamma \beta} \hat{\pi}_{t-1}+\kappa\left[\left(\varphi+\frac{\bar{a}}{\bar{a}-\eta}\right) \hat{Y}_{t}-\frac{\eta}{\bar{a}-\eta} \hat{Y}_{t-1}+\frac{\eta}{\bar{a}-\eta} \hat{a}_{t}-\right. \\
\left.-\left(\frac{\bar{a}}{\bar{a}-\eta}\right)\left(\frac{1}{1-\bar{g}}\right) \hat{g}_{t}+\left(\frac{\eta}{\bar{a}-\eta}\right)\left(\frac{1}{1-\bar{g}}\right) \hat{g}_{t-1}\right]+\hat{u}_{t},
\end{array}
$$

where $\hat{Y}_{t}$ is aggregate output, $\hat{g}_{t}$ represents the government spending-to-output ratio, and $\hat{u}_{t}$ can be interpreted as cost-push shock. The parameters $\beta, \gamma, \varphi$, and $\bar{g}$ are, respectively, the discount factor, the degree of price indexation, the inverse of the Frisch elasticity of labor supply, and the steady-state value of government spending. Furthermore, $\kappa:=\frac{(1-\alpha \beta)(1-\alpha)}{\alpha(1+\varphi \bar{\theta})(1+\gamma \beta)}$. $\alpha$ stands for the degree of price rigidity in the economy and $\bar{\theta}$ for the steady-state value of the elasticity of substitution between intermediate goods.

Monetary policy is characterized by the following rule:

$$
\hat{R}_{t}=\rho_{R} \hat{R}_{t-1}+\left(1-\rho_{R}\right)\left[\phi_{\pi}\left(\hat{\pi}_{t}-\hat{\pi}_{t}^{*}\right)+\phi_{Y}\left(\hat{Y}_{t}-\hat{Y}_{t}^{*}\right)\right]+\epsilon_{R, t}
$$

$\hat{\pi}_{t}^{*}$ is the inflation target and $\hat{Y}_{t}^{*}$ is potential output. The idiosyncratic monetary policy

\footnotetext{
${ }^{2}$ We define the log-linear deviation of a detrended variable from its corresponding steady state as $\hat{X}_{t}=$ $\ln X_{t}-\ln \bar{X}$. Only the fiscal variables $\hat{b}_{t}=b_{t}-\bar{b}, \hat{g}_{t}=g_{t}-\bar{g}, \hat{\tau}_{t}=\tau_{t}-\bar{\tau}$, and $\hat{s}_{t}=s_{t}-\bar{s}$ are normalized by output and linearized around their steady states.
} 
shock $\epsilon_{R, t}$ is assumed to evolve as i.i.d. $N\left(0, \sigma_{R}^{2}\right)$. The parameters $\rho_{R}, \phi_{\pi}$, and $\phi_{Y}$ represent, respectively, interest rate smoothing, responses to deviations of inflation from its target, and responses to deviations of output from its natural level.

The fiscal authority sets lump-sum taxation by a rule:

$$
\hat{\tau}_{t}=\rho_{\tau} \hat{\tau}_{t-1}+\left(1-\rho_{\tau}\right)\left[\psi_{b}\left(\hat{b}_{t-1}-\hat{b}_{t-1}^{*}\right)+\psi_{Y}\left(\hat{Y}_{t}-\hat{Y}_{t}^{*}\right)\right]+\epsilon_{\tau, t}
$$

$\hat{\tau}_{t}$ stands for the tax-revenue-to-output ratio, $\hat{b}_{t}$ is the debt-to-output ratio, and $\hat{b}_{t}^{*}$ is the debt-to-output ratio target. The non-systematic tax policy shock $\epsilon_{\tau, t}$ is assumed to evolve as i.i.d. $N\left(0, \sigma_{\tau}^{2}\right)$. The tax policy rule features tax smoothing $\left(\rho_{\tau}\right)$, systematic reactions of tax revenues to deviations of lagged debt from its target $\left(\psi_{b}\right)$, and to deviations of output from natural output $\left(\psi_{Y}\right)$.

The government spending rule is modeled as

$$
\hat{g}_{t}=\rho_{g} \hat{g}_{t-1}-\left(1-\rho_{g}\right) \chi_{Y}\left(\hat{Y}_{t-1}-\hat{Y}_{t-1}^{*}\right)+\epsilon_{g, t}
$$

$\hat{g}_{t}$ stands for the government spending-to-output ratio. The exogenous shock to government spending $\epsilon_{g, t}$ is assumed to follow an i.i.d.-process with $N\left(0, \sigma_{g}^{2}\right)$. $\rho_{g}$ represents smoothing in government purchases and $\chi_{Y}$ is the response of government spending to the lagged output gap. Under the assumption of flexible prices, the natural level of government spending is:

$$
\hat{g}_{t}^{*}=\rho_{g} \hat{g}_{t-1}^{*}+\epsilon_{g, t}
$$

The government budget constraint is given by:

$$
\hat{b}_{t}=\frac{1}{\beta} \hat{b}_{t-1}+\frac{\bar{b}}{\beta}\left(\hat{R}_{t-1}-\hat{\pi}_{t}-\hat{Y}_{t}+\hat{Y}_{t-1}-\hat{a}_{t}\right)+\hat{g}_{t}-\hat{\tau}_{t}+\hat{s}_{t}
$$

$\hat{s}_{t}$ is the ratio of government transfers to output and the parameter $\bar{b}$ is the steady-state value of the debt-to-output ratio. 
The aggregate resource constraint is given by:

$$
\hat{Y}_{t}=\hat{C}_{t}+\frac{1}{1-\bar{g}} \hat{g}_{t}
$$

The natural level of output is:

$$
\begin{array}{r}
\hat{Y}_{t}^{*}=\frac{\eta}{\varphi(\bar{a}-\eta)+\bar{a}} \hat{Y}_{t-1}^{*}+\frac{\bar{a}}{[\varphi(\bar{a}-\eta)+\bar{a}](1-\bar{g})} \hat{g}_{t}^{*}-\frac{\eta}{[\varphi(\bar{a}-\eta)+\bar{a}](1-\bar{g})} \hat{g}_{t-1}^{*}- \\
-\frac{\eta}{\varphi(\bar{a}-\eta)+\bar{a}} \hat{a}_{t} .
\end{array}
$$

Finally, six additional exogenous shocks drive economic fluctuations. They are all assumed to evolve according to univariate $\mathrm{AR}(1)$ processes.

Preferences evolve as

$$
\hat{d}_{t}=\rho_{d} \hat{d}_{t-1}+\epsilon_{d, t} \quad \text { with } \epsilon_{d, t} \sim \text { i.i.d. } N\left(0, \sigma_{d}^{2}\right)
$$

Technology evolves as

$$
\hat{a}_{t}=\rho_{a} \hat{a}_{t-1}+\epsilon_{a, t} \quad \text { with } \epsilon_{a, t} \sim \text { i.i.d. } N\left(0, \sigma_{a}^{2}\right) .
$$

Markup shocks are assumed to follow

$$
\hat{u}_{t}=\rho_{u} \hat{u}_{t-1}+\epsilon_{u, t} \quad \text { with } \epsilon_{u, t} \sim \text { i.i.d. } N\left(0, \sigma_{u}^{2}\right) .
$$

Government transfers are given by

$$
\hat{s}_{t}=\rho_{s} \hat{s}_{t-1}+\epsilon_{s, t} \quad \text { with } \epsilon_{s, t} \sim \text { i.i.d. } N\left(0, \sigma_{s}^{2}\right) .
$$

The inflation target evolves as

$$
\hat{\pi}_{t}^{*}=\rho_{\pi} \hat{\pi}_{t-1}^{*}+\epsilon_{\pi, t} \quad \text { with } \epsilon_{\pi, t} \sim \text { i.i.d. } N\left(0, \sigma_{\pi}^{2}\right) .
$$


The debt-to-output ratio target follows

$$
\hat{b}_{t}^{*}=\rho_{b} \hat{b}_{t-1}^{*}+\epsilon_{b, t} \quad \text { with } \epsilon_{b, t} \sim \text { i.i.d. } N\left(0, \sigma_{b}^{2}\right) .
$$

\subsection{Model solution under different policy regimes}

A unique equilibrium of the economy arises if either monetary policy is active while fiscal policy is passive (regime M) or monetary policy is passive while fiscal policy is active (regime F). If both monetary and fiscal policy are passive multiple equilibria exist (indeterminacy). No stationary equilibrium exists if both authorities act actively (explosiveness). The boundaries of the four distinct policy regimes can be characterized analytically in Bhattarai et al. (2016)'s model. In particular, monetary policy is active if

$$
\phi_{\pi}>1-\phi_{Y}\left(\frac{1-\tilde{\beta}}{\tilde{\kappa}}\right)
$$

where $\tilde{\beta}=\frac{\gamma+\beta}{1+\gamma \beta}$ and $\tilde{\kappa}=\frac{(1-\alpha \beta)(1-\alpha)}{\alpha(1+\varphi \bar{\theta})(1+\gamma \beta)}\left(1+\varphi+\frac{\chi_{Y}}{1-\bar{g}}\right)$, while fiscal policy is active if

$$
\psi_{b}<\frac{1}{\beta}-1
$$

We collect the parameters of the loglinearized model in the vector $\vartheta$ with domain $\Theta$ and solve the system of equations for its state-space representation. ${ }^{3}$ Under determinacy (regime F, regime M), we employ the solution algorithm for linear rational expectations models of Sims (2002) which expresses the model solution as

$$
z_{t}=\Gamma_{1}^{*}(\vartheta) z_{t-1}+\Psi^{*}(\vartheta) \epsilon_{t}
$$

where $z_{t}$ is a vector of state variables, $\epsilon_{t}$ is a vector of exogenous variables, while both $\Gamma_{1}^{*}$ and $\Psi^{*}$ are coefficient matrices that depend on the model parameters collected in the vector

\footnotetext{
${ }^{3}$ More details on the implementation of the model solution are given in Appendix A.
} 
$\vartheta$. Under indeterminacy, we apply the generalization of this procedure suggested by Lubik and Schorfheide (2003, 2004):

$$
z_{t}=\Gamma_{1}^{*}(\vartheta) z_{t-1}+\left[\Gamma_{0, \epsilon}^{*}(\vartheta)+\Gamma_{0, \zeta}^{*}(\vartheta) \tilde{M}\right] \epsilon_{t}+\Gamma_{0, \zeta}^{*}(\vartheta) M_{\zeta} \zeta_{t} .
$$

Under indeterminacy, the transmission of fundamental shocks $\epsilon_{t}$ is no longer uniquely determined as it depends not only on the coefficient matrix $\Gamma_{0, \epsilon}^{*}$, but also on the matrices $\tilde{M}$ and $\Gamma_{0, \zeta}^{*}$. Second, an exogenous sunspot shock $\zeta_{t}$, unrelated to the fundamental shocks $\epsilon_{t}$, potentially affects the dynamics of the model variables $z_{t}$. This effect depends on the coefficient matrices $\Gamma_{0, \zeta}^{*}$ and $M_{\zeta}$.

\section{$3 \quad$ Estimation strategy - rethinking the status quo}

In this section, we present the empirical estimation of the model. Not only do we describe the prior distributions and the dataset, we also illustrate the procedure for posterior sampling we choose that makes our study distinct. The existing empirical studies that aim to determine the prevailing monetary-fiscal policy mix in a DSGE model, rely exclusively on the RWMH algorithm for posterior sampling. ${ }^{4}$ One caveat of the RWMH sampler is that its efficiency deteriorates as the DSGE model increases in size. The RWMH sampler is inherently serial and, hence, very slowly to converge. It can also get stuck at a local mode and, hence, fails to explore the parameter space in its correct proportions. To circumvent this drawback, the literature adopts the following approach. First, the model's parameter space is restricted to impose a distinct policy regime (regime F, regime M, or indeterminacy). Second, each of these regimes is treated as a distinct model and is estimated separately with the RWMH. Last, the best fitting is determined by a model selection criterion, usually marginal data densities (MDD).

\footnotetext{
${ }^{4}$ In short, the RWMH is an iterative simulator that belongs to the class of MCMC techniques. Herbst and Schorfheide (2015), for instance, explain the sampler in detail.
} 
Due to the model's size and complexity, applying the RWMH over the entire parameter space in one step is simply not feasible in our application. In comparison, our preferred posterior sampler, the SMC algorithm, sends out many thousand particles at the same time to explore the entire parameter space. As these particles are independent of each other, the SMC can take advantage of parallelization. All these features make the SMC sampler the more efficient choice for models with multiple regimes.

\subsection{Prior distributions and calibrated parameters}

In line with Bhattarai et al. (2016), we fix a few model parameters. We calibrate the inverse of the Frisch elasticity of labor supply to $\varphi=1$ and the steady-state value of the elasticity of substitution between goods to $\bar{\theta}=8$, since they cannot be separately identified from the Calvo parameter $\alpha$. We also fix the parameters measuring the persistence of the time-varying policy targets to $\rho_{\pi}=\rho_{b}=0.995$.

Table 1: Prior distributions

\begin{tabular}{|c|c|c|c|c|c|}
\hline \multirow[b]{2}{*}{ Parameter } & \multicolumn{5}{|c|}{ Prior } \\
\hline & Range & Distribution & Mean & SD & 90 percent int. \\
\hline \multicolumn{6}{|l|}{ Monetary policy } \\
\hline$\phi_{\pi}$, interest rate response to inflation & $\mathbb{R}^{+}$ & $\mathrm{N}$ & 0.8 & 0.6 & {$[0.14,1.84]$} \\
\hline$\phi_{Y}$, interest rate response to output & $\mathbb{R}^{+}$ & $\mathrm{G}$ & 0.3 & 0.1 & {$[0.16,0.5]$} \\
\hline$\rho_{R}$, response to lagged interest rate & {$[0,1)$} & $\mathrm{B}$ & 0.6 & 0.2 & {$[0.24,0.9]$} \\
\hline \multicolumn{6}{|l|}{ Fiscal policy } \\
\hline$\psi_{b}$, tax response to lagged debt & $\mathbb{R}$ & $\mathrm{N}$ & 0 & 0.1 & {$[-0.16,0.16]$} \\
\hline$\psi_{Y}$, tax response to output & $\mathbb{R}$ & $\mathrm{N}$ & 0.4 & 0.3 & {$[-0.1,0.9]$} \\
\hline $\begin{array}{l}\chi_{Y}, \text { govt spending response to } \\
\text { lagged output }\end{array}$ & $\mathbb{R}$ & $\mathrm{N}$ & 0.4 & 0.3 & {$[-0.1,0.9]$} \\
\hline$\rho_{g}$, response to lagged govt spending & {$[0,1)$} & $\mathrm{B}$ & 0.6 & 0.2 & {$[0.24,0.9]$} \\
\hline$\rho_{\tau}$, response to lagged taxes & {$[0,1)$} & $\mathrm{B}$ & 0.6 & 0.2 & {$[0.24,0.9]$} \\
\hline \multicolumn{6}{|l|}{ Preference and $\mathrm{HHs}$} \\
\hline$\eta$, habit formation & {$[0,1)$} & $\mathrm{B}$ & 0.5 & 0.2 & {$[0.17,0.83]$} \\
\hline$\mu:=100\left(\beta^{-1}-1\right)$, discount factor & $\mathbb{R}^{+}$ & $\mathrm{G}$ & 0.25 & 0.1 & {$[0.11,0.44]$} \\
\hline \multicolumn{6}{|l|}{ Frictions } \\
\hline$\alpha$, price stickiness & {$[0,1)$} & $\mathrm{B}$ & 0.5 & 0.2 & {$[0.17,0.83]$} \\
\hline
\end{tabular}


Table 1: Prior distributions - continued

\begin{tabular}{lccccc}
\hline \hline & \multicolumn{5}{c}{ Prior } \\
\cline { 2 - 6 } \multicolumn{1}{c}{ Parameter } & Range & Distribution & Mean & SD & 90 percent int. \\
\hline$\gamma$, price indexation & {$[0,1)$} & $\mathrm{B}$ & 0.6 & 0.2 & {$[0.24,0.9]$} \\
Shocks & & & & & \\
$\rho_{d}$, preference & {$[0,1)$} & $\mathrm{B}$ & 0.6 & 0.2 & {$[0.24,0.9]$} \\
$\rho_{a}$, technology & {$[0,1)$} & $\mathrm{B}$ & 0.4 & 0.2 & {$[0.1,0.76]$} \\
$\rho_{u}$, cost-push & {$[0,1)$} & $\mathrm{B}$ & 0.6 & 0.2 & {$[0.24,0.9]$} \\
$\rho_{s}$, transfers & {$[0,1)$} & $\mathrm{B}$ & 0.6 & 0.2 & {$[0.24,0.9]$} \\
$\sigma_{g}$, govt spending & $\mathbb{R}^{+}$ & Inv. Gamma & 0.1 & 4 & {$[0.65,0.24]$} \\
$\sigma_{d}$, preference & $\mathbb{R}^{+}$ & Inv. Gamma & 0.3 & 4 & {$[0.19,0.72]$} \\
$\sigma_{a}$, technology & $\mathbb{R}^{+}$ & Inv. Gamma & 0.5 & 4 & {$[0.32,1.17]$} \\
$\sigma_{u}$, cost-push & $\mathbb{R}^{+}$ & Inv. Gamma & 0.04 & 4 & {$[0.026,0.094]$} \\
$\sigma_{s}$, transfers & $\mathbb{R}^{+}$ & Inv. Gamma & 0.08 & 4 & {$[0.052,0.188]$} \\
$\sigma_{R}$, monetary policy & $\mathbb{R}^{+}$ & Inv. Gamma & 0.15 & 4 & {$[0.098,0.353]$} \\
$\sigma_{\tau}$, tax & $\mathbb{R}^{+}$ & Inv. Gamma & 0.2 & 4 & {$[0.13,0.48]$} \\
$\sigma_{\pi}$, inflation target & $\mathbb{R}^{+}$ & Inv. Gamma & 0.003 & 4 & $[0.002,0.007]]$ \\
$\sigma_{b}$, debt/output target & $\mathbb{R}^{+}$ & Inv. Gamma & 0.05 & 4 & {$[0.033,0.118]$}
\end{tabular}

Steady state

$a:=100(\bar{a}-1)$, technology

$\begin{array}{lcccc}\mathbb{R} & \mathrm{N} & 0.55 & 0.1 & {[0.38,0.71]} \\ \mathbb{R} & \mathrm{N} & 0.8 & 0.1 & {[0.63,0.96]} \\ \mathbb{R} & \mathrm{N} & 35 & 2 & {[31.71,38.3]} \\ \mathbb{R} & \mathrm{N} & 25 & 2 & {[21.73,28.27]} \\ \mathbb{R} & \mathrm{N} & 22 & 2 & {[18.81,25.31]}\end{array}$

Indeterminacy

$\sigma_{\zeta}$, sunspot shock

$M_{g \zeta}$

$M_{d \zeta}$

$M_{a \zeta}$

$M_{u \zeta}$

$M_{s \zeta}$

$M_{R \zeta}$

$M_{\tau \zeta}$

$M_{\pi \zeta}$

$M_{b \zeta}$

\begin{tabular}{ccccc}
$\mathbb{R}^{+}$ & Inv. Gamma & 0.2 & 4 & {$[0.13,0.48]$} \\
$\mathbb{R}$ & $\mathrm{N}$ & 0 & 1 & {$[-1.64,1.64]$} \\
$\mathbb{R}$ & $\mathrm{N}$ & 0 & 1 & {$[-1.64,1.64]$} \\
$\mathbb{R}$ & $\mathrm{N}$ & 0 & 1 & {$[-1.64,1.64]$} \\
$\mathbb{R}$ & $\mathrm{N}$ & 0 & 1 & {$[-1.64,1.64]$} \\
$\mathbb{R}$ & $\mathrm{N}$ & 0 & 1 & {$[-1.64,1.64]$} \\
$\mathbb{R}$ & $\mathrm{N}$ & 0 & 1 & {$[-1.64,1.64]$} \\
$\mathbb{R}$ & $\mathrm{N}$ & 0 & 1 & {$[-1.64,1.64]$} \\
$\mathbb{R}$ & $\mathrm{N}$ & 0 & 1 & {$[-1.64,1.64]$} \\
$\mathbb{R}$ & $\mathrm{N}$ & 0 & 1 & {$[-1.64,1.64]$} \\
\hline
\end{tabular}

Note: The Inverse Gamma prior distributions have the form $p(x \mid \nu, s) \propto x^{-\nu-1} e^{-\nu s^{2} / 2 x^{2}}$, where $\nu=4$ and $s$ is given by the value in the column denoted as "Mean".

Table 1 specifies the prior distributions. They extend over a broad range of parameter 
values. As we initialize the SMC algorithm from the prior, we carefully tailored a prior that results in realistic model implications, but nevertheless remains agnostic about the prevailing policy regime. ${ }^{5}$ In the following, we discuss only the key parameters of our analysis.

Specifically, the policy parameters in the monetary and fiscal policy rule $\phi_{\pi}$ and $\psi_{b}$ play a central role in our analysis as they determine the policy regime. For $\phi_{\pi}$, we choose a Normal distribution restricted to the positive domain with an implied $90 \%$ probability interval from 0.14 to 1.84 , while for $\psi_{b}$ the interval extends from -0.16 to 0.16 . Our choice is motivated by the consideration to construct prior distributions that yield more or less equal probabilities for regime $\mathrm{F}$ and indeterminacy. In particular, as we initialize the SMC algorithm from the prior, we do not want to impose artificially a certain policy regime before confronting the model with the data. The implied prior probabilities of the policy regimes presented in Table 2 support our choice. Regime $\mathrm{F}$ and the indeterminacy regime receive almost identical support.

Table 2: Prior probability of pre-Volcker policy regimes

\begin{tabular}{lcccc}
\hline \hline & Regime M & Regime F & Indeterminacy & Explosiveness \\
\hline Probability & 20.27 & 29.94 & 28.83 & 20.96 \\
\hline
\end{tabular}

Note: The prior probabilities of the policy regimes are obtained from a prior predictive analysis. We drew $\vartheta 20,000$ times from the priors specified in Table 1, solved the model with each draw and computed the shares of each policy regime.

A second group of parameters we want to discuss are those necessary to characterize the indeterminacy model solution. For the parameters in the matrix $M$, representing agents' self-fulfilling beliefs, we choose priors centered around zero in order to let the data decide if and how indeterminacy changes the propagation mechanism of the fundamental shocks. The prior of the sunspot shock's standard deviation $\sigma_{\zeta}$ is also identical to Lubik and Schorfheide

\footnotetext{
${ }^{5}$ In Appendix B we show results from a prior predictive analysis. In particular, we take 20,000 draws from the prior, simulate the model's observables and plot these simulated time series against the actual data from 1960:Q1 to 1979:Q2 that we use for estimating the model.
} 
(2004).

\subsection{Data}

We use the dataset of Bhattarai et al. (2016). ${ }^{6}$ We fit the loglinearized DSGE model to six quarterly U.S. time series and estimate the model for the pre-Volcker sample 1960:Q1 to 1979:Q2. The list of observables includes output, inflation, nominal interest rates, the tax-revenue-to-output ratio, the market value of the government debt-to-output ratio, and the government spending-to-output ratio.

\subsection{Sequential Monte Carlo posterior sampling}

We calculate the likelihood function of the model solution over the determinacy and indeterminacy region of the parameter space as

$$
p(Y \mid \vartheta, M)=\left\{\vartheta \in \Theta^{D}\right\} p^{D}(Y \mid \vartheta)+\left\{\vartheta \in \Theta^{I}\right\} p^{I}(Y \mid \vartheta, M)
$$

where $Y$ is the relevant data and $h(x)=\{x \in \mathfrak{X}\}$ is the indicator function that is one if $x \in \mathfrak{X}$ and zero otherwise. We are interested in the posterior density $p(\vartheta, M \mid Y)$, which is given by

$$
p(\vartheta, M \mid Y)=\frac{p(Y \mid \vartheta, M) p(\vartheta, M)}{p(Y)}, \text { where } p(Y)=\int p(Y \mid \vartheta, M) p(\vartheta, M) d \vartheta d M
$$

$p(\vartheta, M)$ stands for the prior density and $p(Y)$ represents the marginal data density.

Posterior inference in DSGE models relies on sampling techniques as the moments of $p(\vartheta, M \mid Y)$ cannot be characterized in closed forms. For our application, we choose the SMC algorithm introduced to the DSGE literature by Creal (2007) then further enhanced and

\footnotetext{
${ }^{6}$ The dataset is downloadable from the supplemental material of their study https://dataverse. harvard.edu/dataset. xhtml?persistentId=doi:10.7910/DVN/OHUWKM. More details on the data and the corresponding measurement equations are given in Appendix C.
} 
theoretically justified by Herbst and Schorfheide $(2014,2015) .{ }^{7}$ Three considerations make the SMC algorithm our preferred choice. First, the SMC is able to deal with difficult posterior surfaces, an outcome that a priori cannot be excluded in the case of the DSGE model with monetary-fiscal policy interactions. Due to this feature, neither are we are obliged to estimate the model separately nor must we compare model fit across regimes. Rather, we let the SMC algorithm explore the entire parameter space such that the probability of each policy regime is directly determined by the data. Second, the SMC is amenable to parallelization and, thus computationally attractive, especially for larger DSGE models. Third, the SMC can be easily initialized by taking independent draws from the prior density. A time-consuming search for a mode is not necessary.

The basic concept of the SMC relies on importance sampling, which means that the posterior $p(\vartheta, M \mid Y)$ is approximated by an easy-to-sample proposal, or source density. However, in the high-dimensional parameter space of DSGE models, good proposal densities are difficult to obtain. That is why the SMC constructs proposal densities sequentially. More precisely, the algorithm draws from a sequence of bridge densities that link a known starting distribution with the targeted posterior density. A meaningful starting distribution constitutes the prior $p(\vartheta, M)$. The bridge distributions, in contrast, differ in the amount of information from the likelihood they contain. At each stage of the algorithm, an increment of the likelihood is added to the proposal density. At the moment the full information from the likelihood has been released, an approximation of the posterior is obtained. In particular, the sequence of $n$ distributions is given by

$$
p_{n}(\vartheta, M \mid Y)=\frac{[p(Y \mid \vartheta, M)]^{\delta_{n}} p(\vartheta, M)}{\int[p(Y \mid \vartheta, M)]^{\delta_{n}} p(\vartheta, M) d \vartheta d M}, \quad n=1, \ldots, N_{\delta}
$$

We follow Herbst and Schorfheide (2014) and choose the tuning parameter $\delta_{n}$ as an increasing sequence of values such that $\delta_{1}=0$ and $\delta_{N_{\delta}}=1$. The length of this sequence coincides

\footnotetext{
${ }^{7}$ Chopin (2002), Del Moral et al. (2006), and Creal (2012), among others, provide further details on SMC algorithms.
} 
with the number of importance samplers. At the first stage of the algorithm, $p_{1}(\vartheta, M \mid Y)$ is the prior density $p(\vartheta, M)$. At the last stage, the final proposal density $p_{N_{\delta}}(\vartheta, M \mid Y)$ constitutes the posterior $p(\vartheta, M \mid Y)$. In particular, our tempering schedule $\left\{\delta_{n}\right\}_{n=1}^{N_{\delta}}$ is given by $\delta_{n}=\left(n-1 / N_{\delta}-1\right)^{\lambda}$. The tuning parameter $\lambda$ determines how much information from the likelihood is incorporated in each proposal density.

In a nutshell, the SMC draws in $N_{\delta}$ stages sequentially $N$ parameter vectors $\vartheta^{i}, i=$ $1, \ldots, N$ from the proposal densities and assigns them with importance weights $\tilde{W}^{i}$. Each of the $i$ pairs $\left(\vartheta^{i}, \tilde{W}^{i}\right)$ is known as a particle, and the set of particles $\left\{\left(\vartheta^{i}, \tilde{W}^{i}\right)\right\}_{i=1}^{N}$ approximates the density in iteration. Each stage of the SMC consists of three steps. First, in the correction step of stage $n$, the particles of the previous stage $\left\{\left(\vartheta_{n-1}^{i}, \tilde{W}_{n-1}^{i}\right)\right\}_{i=1}^{N}$ are reweighted to correct for the difference between $p_{n-1}(\vartheta, M \mid Y)$ and $p_{n}(\vartheta, M \mid Y)$. The second step, the selection step, controls the accuracy of the particle approximation. Whenever the distribution of weights becomes too uneven, systematic resampling restores a well-balanced set of particles. In the last step, the mutation step, the particle values are propagated around in the parameter space by $M_{M H}$ iterations of a RWMH algorithm with $N_{\text {blocks }}$ random blocks. The particles' new location determines the updated density $p_{n}(\vartheta, M \mid Y) .{ }^{8}$

To estimate the model, we choose the following tuning parameters for the SMC. We use $N=20,000$ particles, $N_{\delta}=600$ stages, $\lambda=2.4, N_{\text {blocks }}=10, M_{M H}=2$. As suggested by Herbst and Schorfheide (2014), $\lambda$ is determined by examining the particle degeneracy after the first piece of information of the likelihood was added to the prior density in $n=1$. We increased $\lambda$ until at least $80 \%$ of the total number of particles $(16,000)$ was retained. To choose $N_{\text {blocks }}$ and $M_{M H}$, we monitored the acceptance rate in the mutation step in preliminary runs. $\quad N_{\text {blocks }}=10$ and $M_{M H}=2$ ensured a stable acceptance rate of $25 \%$ without down-scaling the proposal variance too much.

\footnotetext{
${ }^{8} \mathrm{~A}$ more detailed description of the SMC algorithm is given in Appendix D.
} 


\section{The monetary-fiscal policy mix in the pre-Volcker period}

In this section, we summarize the estimation results and determine the monetary-fiscal policy mix in the pre-Volcker period. We show two sets of estimation results. First, we show results of estimations in which we restrict the parameter space and apply SMC sampling to estimate each policy regime sequentially. The purpose of this exercise is to show (i) that the SMC sampler is able to replicate the RWMH estimation results of Bhattarai et al. (2016), our reference study, and (ii) that our prior specification does not affect the probability of policy regimes in the posterior. Hence, potential differences in findings are driven neither by the prior specification nor the sampling technique, but rather induced by restricting or not restricting the parameter space. In a second step, we show and discuss the results of the unrestricted estimation, our preferred approach. In the final discussion, we argue that the SMC approach is able to reconcile the empirical findings of the fixed-regime and regimeswitching DSGE model literature, while also providing some intuition why restricting or not restricting the parameter space during estimation matters.

\subsection{Restricted estimation}

\section{Prior distribution as in Bhattarai et al. (2016)}

To understand how changing the posterior sampler influences the estimation results, we apply the SMC algorithm and replicate, in a first step, the study of Bhattarai et al. (2016). For this exercise, we follow strictly the approach of Bhattarai et al. (2016). We use the same dataset, and the same prior distributions. ${ }^{9}$ Only in terms of posterior sampling, we do not rely on RWMH sampling, but apply the SMC algorithm instead. We restrict the parameter space and estimate each policy regime 50 times with the SMC sampler.

\footnotetext{
${ }^{9}$ Appendix E contains the results of a prior predictive analysis for the prior specification of Bhattarai et al. (2016). For details on this prior specification, we refer the reader to the Online Appendix of the original study.
} 
Looking at the estimated marginal data densities of each regime, presented in Table 3, we come to the same conclusion as Bhattarai et al. (2016): the U.S.-economy in the pre-Volcker period was in the indeterminacy regime. In this estimation, regime $\mathrm{F}$ and regime $\mathrm{M}$ receive no support from the data. ${ }^{10}$

Table 3: Log marginal data densities for each policy regime from restricted estimation

\begin{tabular}{cccc}
\hline & Regime M & Regime F & Indeterminacy \\
\hline Log MDD & -541.85 & -537.54 & -521.41
\end{tabular}

Note: The log marginal data density is obtained as a byproduct during the correction step of the SMC algorithm, see Herbst and Schorfheide (2014) for further details. For each regime, its mean is computed over 50 independent runs of the SMC algorithm.

Figure 1 shows plots of the posterior densities of the policy parameters for regime $\mathrm{F}$ and the indeterminacy regime. ${ }^{11}$ The mean estimates for the Taylor-coefficient $\phi_{\pi}$ (regime F: 0.71; indeterminacy: 0.31) and $\psi_{b}$ (regime F: -0.08; indeterminacy: 0.05) are in line with the findings of Bhattarai et al. (2016). Hence, using the SMC instead of the RWMH algorithm for posterior sampling does not influence the estimation results.

\footnotetext{
${ }^{10}$ The finding that monetary policy in the pre-Volcker period was mainly passive, is widely established in the literature. Therefore, in the following, we focus our discussion entirely on the still open role of fiscal policy and look exclusively on regime $\mathrm{F}$ and the indeterminacy regime.

${ }^{11}$ Appendix F.1 contains density plots of the remaining parameters as well al tables with estimated means, standard deviations, and credible bands for all parameters. It also incorporates the configuration details of the SMC algorithm for the restricted estimation. To make the results of the restricted estimation more comparable to the unrestricted estimation, we transformed the policy parameters $\phi_{\pi}^{*}$ and $\psi_{b}^{*}$ to $\phi_{\pi}$ and $\psi_{b}$.
} 

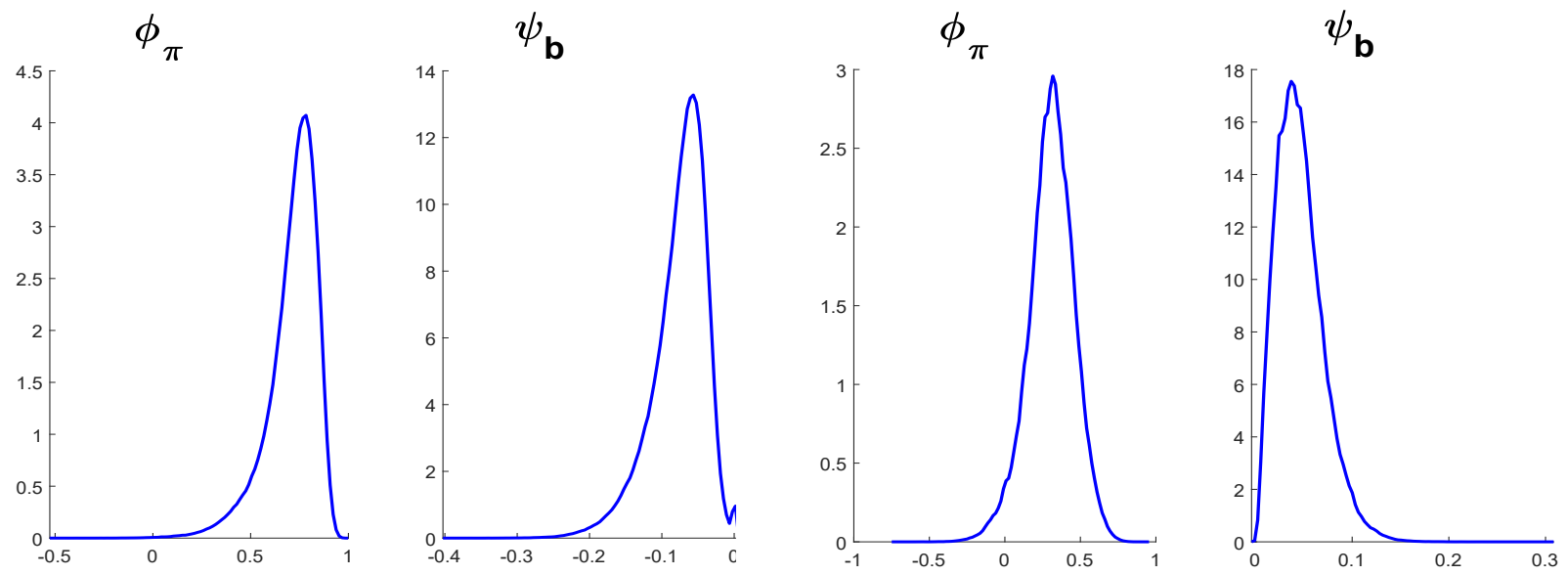

(a) Regime F

(b) Indeterminacy

Figure 1: Posterior densities of the policy parameters $\phi_{\pi}$ and $\psi_{b}$ for regime $\mathrm{F}$ and the indeterminacy regime.

\section{Prior distribution as in Section 3.1}

In a next step, we conduct the restricted SMC estimation with the prior specification as outlined in Table 1. One exception is the prior specifications for the policy parameters $\phi_{\pi}$ and $\psi_{b}$. To ensure that we completely impose a particular policy regime during estimation, we follow again Bhattarai et al. (2016) and estimate the model with the reparametrized policy parameters $\phi_{\pi}^{*}$ and $\psi_{b}^{*}{ }^{12}$

Table 4 shows the estimated marginal data densities of each regime. Also, with our preferred prior specification, we come to the conclusion that in the U.S. in the pre-Volcker period the indeterminacy regime receives the best support from the data.

\footnotetext{
${ }^{12}$ Appendix E contains the prior specification of the reparametrized policy parameters.
} 
Table 4: Log marginal data densities for each policy regime from restricted estimation

\begin{tabular}{lccc}
\hline \hline & Regime M & Regime F & Indeterminacy \\
\hline Log MDD & -548.72 & -542.72 & -523.17
\end{tabular}

Note: The log marginal data density is obtained as a byproduct during the correction step of the SMC algorithm, see Herbst and Schorfheide (2014) for further details. For each regime, its mean is computed over 50 independent runs of the SMC algorithm.

Figure 2 shows plots of the posterior densities of the policy parameters for regime $\mathrm{F}$ and the indeterminacy regime. ${ }^{13}$ The shapes of the posterior densities are comparable to the findings in the previous subsection. The mean estimates for the Taylor-coefficient $\phi_{\pi}$ (regime F: 0.54; indeterminacy: 0.11 ) and $\psi_{b}$ (regime F: -0.02; indeterminacy: 0.05) change only slightly. Hence, using our preferred prior specification together with SMC posterior sampling does not influence the estimation results.

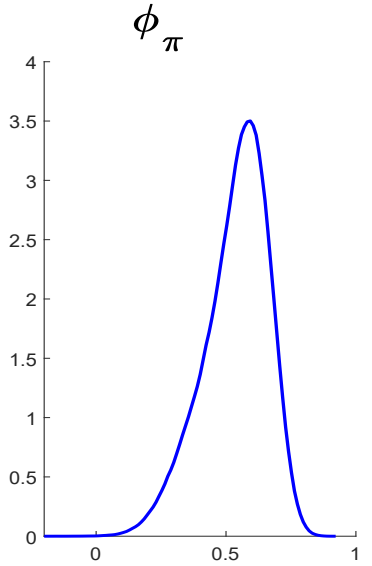

(a) Regime F
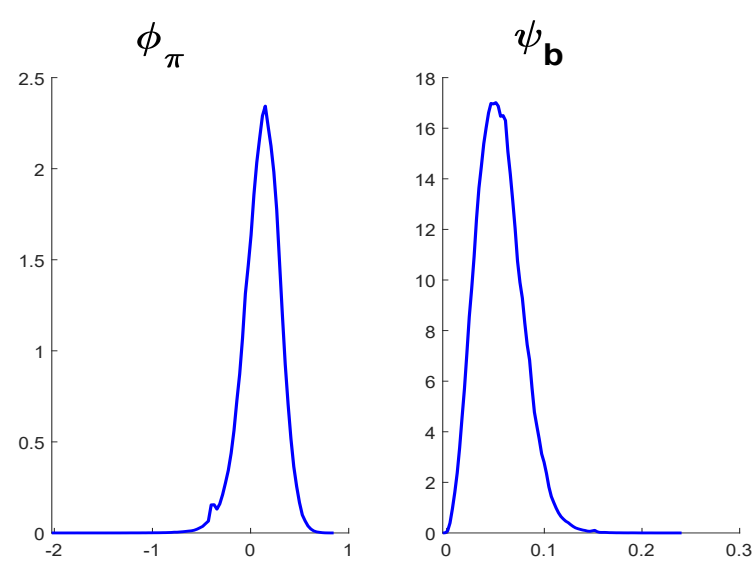

(b) Indeterminacy

Figure 2: Posterior densities of the policy parameters $\phi_{\pi}$ and $\psi_{b}$ for regime $\mathrm{F}$ and the indeterminacy regime.

\footnotetext{
${ }^{13}$ Appendix F.1 contains density plots of the remaining parameters, as well as tables with estimated means, standard deviations, and credible bands for all parameters. It also contains the configuration details of the SMC algorithm for the restricted estimation. To make the results of the restricted estimation more comparable to the unrestricted estimation, we transformed the policy parameters $\phi_{\pi}^{*}$ and $\psi_{b}^{*}$ to $\phi_{\pi}$ and $\psi_{b}$.
} 


\subsection{Unrestricted estimation}
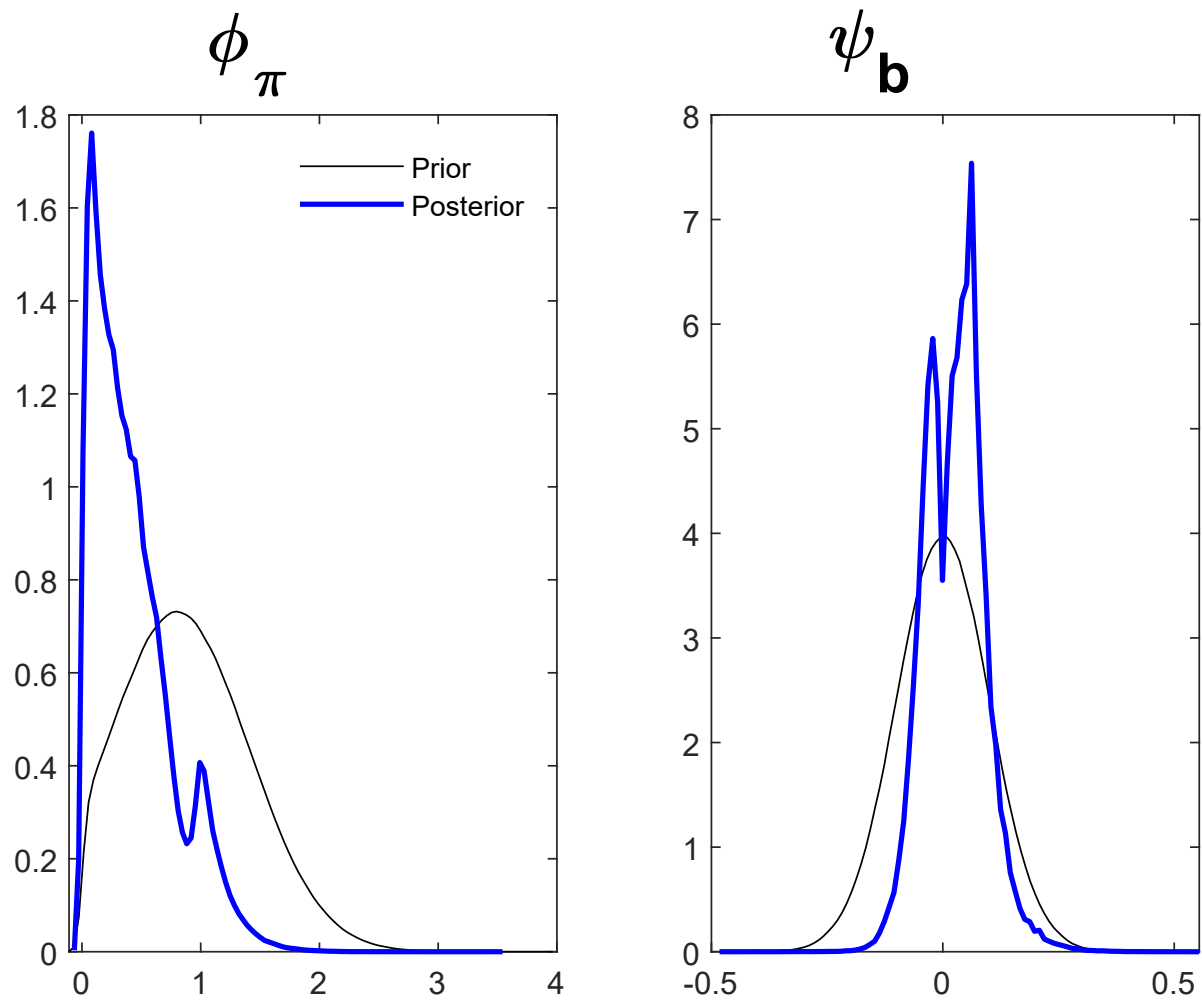

Figure 3: Prior and posterior densities of the policy parameters from the unrestricted estimation. The blue bold line depicts the posterior density, the black line the prior density.

Figure 3 shows prior and posterior density plots of the estimated policy parameters from the unrestricted estimation. ${ }^{14}$ Estimating the model over its entire parameter space clearly changes the picture. The posterior densities of $\phi_{\pi}$ and $\psi_{b}$ display pronounced bimodalities around the policy regimes. For $\phi_{\pi}$, the mode to the left of one corresponds to a passive monetary authority, while the mode on the right corresponds to an active central bank. The boundary of fiscal policy $\left(\psi_{b}\right)$ lies around zero. The mode to the left of the boundary corresponds to an active fiscal authority, the mode to the right to a passive fiscal authority. It is also noticeable that the probability mass below each mode is unequally distributed.

To shed more light on the estimated monetary-fiscal policy mix, we present the posterior

\footnotetext{
${ }^{14}$ Appendix F.2 contains the density plots of the remaining parameters as well as tables with estimated means, standard deviations, and credible bands for all parameters.
} 
probabilities of the policy regimes in the pre-Volcker period. As Table 5 shows, the dominant regime in the pre-Volcker period is, at $43.54 \%$, the indeterminacy regime. However, with $36.81 \%$ probability, regime F scores only slightly worse. Regime M receives the least support from the data, at $19.65 \%{ }^{15}$

Table 5: Posterior probability of pre-Volcker policy regimes

\begin{tabular}{cccc}
\hline \hline & Regime M & Regime F & Indeterminacy \\
\hline Probability & 19.65 & 36.81 & 43.54 \\
\hline
\end{tabular}

Note: To obtain the posterior probabilities, we solved the model with each of the 20,000 particles and computed the shares of each policy regime over 50 independent runs of the SMC algorithm.

\subsection{Discussion}

Although the unrestricted estimation in Section 4.2 confirms, on average, the finding of Bhattarai et al. (2016) that indeterminacy is the most likely regime pre-Volcker, we argue in the following that regime $\mathrm{F}$ also matters for the macroeconomic dynamics in the pre-Volcker period. First, in our analysis, regime F receives, at $36.81 \%$, considerable probability that is only seven percentage points less than the, on average, dominant indeterminacy regime. Due to this significant empirical support, regime F should not simply be neglected. Second, many existing studies already convincingly discuss quantitative or narrative evidence for a leading fiscal authority during particular periods in the pre-Volcker era. Sims (2011), for instance, refers to the emerging primary deficits in the U.S. related to President Ford's tax cuts and rebates in 1975. Bianchi and Ilut (2017), in a regime-switching DSGE model, even provide empirical evidence for fiscal dominance in the U.S. during the 1960s and 1970s, outlining the

\footnotetext{
${ }^{15}$ The finding that regime $\mathrm{M}$ receives some support in the data is in line with the findings of the regimeswitching DSGE model literature. For instance, Bianchi (2012) finds that an active monetary policy regime was in place in the early 1960s. As we estimate the model over the sample 1960:Q1 to 1979:Q2, we pick up this period.
} 
fiscal expansion due to the Vietnam War and Lyndon B. Johnson's Great Society reforms. ${ }^{16}$ Neglecting this evidence is not constructive for the debate.

Our chosen SMC approach's merit is that it can create new perspectives in a fixed-regime model environment. As we can estimate the model over its entire parameter space, we remain agnostic and strictly let the data determine each policy regime's probability. In contrast, in our application, RWMH sampling works only for a subset of the parameter space and, hence, would force us to take a zero-one decision. After having estimated each policy regime and its corresponding MDD separately, we would conclude that the indeterminacy regime receives the best support by the data. The other policy regimes would not be considered. Instead, our analysis allows us to draw a more nuanced conclusion: although indeterminacy was the dominant regime throughout the 1960:Q1 to 1979:Q2 sample, regime F also mattered.

Why do the results from the restricted and the unrestricted estimation bring us to different conclusions? Section 4.1 shows that our main result is driven neither by the sampling algorithm per se nor our prior specification. The only difference of our study compared to Bhattarai et al. (2016) is that employing the SMC allows us to estimate the model over its continuous parameter space. A model comparison of different, discrete regimes is not necessary as the policy regimes' probabilities are directly determined during estimation.

The fact that Bayesian model comparison methods suffer from limitations is, for instance, discussed by Sims (2003) and Gelman et al. (2013). The authors argue that Bayesian model comparison results must be treated with caution when a discrete collection of models is used as a proxy for an actual continuous parameter space. The results from the unrestricted estimation, as well as the findings of the regime-switching literature, however, imply that, pre-Volcker, all three policy regimes were in place, although of varying durations. This suggests that the assumption of a continuous parameter space, is, in our application, more suitable than estimating and comparing three discrete models.

\footnotetext{
${ }^{16}$ Further references that provide evidence for fiscal dominance in the U.S. in the pre-Volcker period include, among others, Davig and Leeper (2006), Bianchi (2012), or Chen et al. (2019). All these studies employ regime-switching model frameworks.
} 


\section{Revisiting the Great Inflation}

The estimation in the previous section shows that the macroeconomic dynamics in the preVolcker period are similarly driven by indeterminacy and fiscal dominance. In the light of these results, we revisit one of the most pressing macroeconomic questions of this episode, namely, what caused the Great Inflation. In a first step, we use our findings to carry out a historical shock decomposition of pre-Volcker inflation. In a second step, we conduct a counterfactual analysis to quantify the importance of fiscal policy actions in the run up of inflation.

\subsection{Shock decomposition}

We partition the draws from the posterior according to the corresponding policy regimes and conduct the historical decomposition for indeterminacy and regime F separately.

Figure 4 shows the results for the indeterminacy regime. In the indeterminacy regime, pre-Volcker inflation was mainly driven by non-policy shocks, in particular, preference, markup, and technology shocks. 


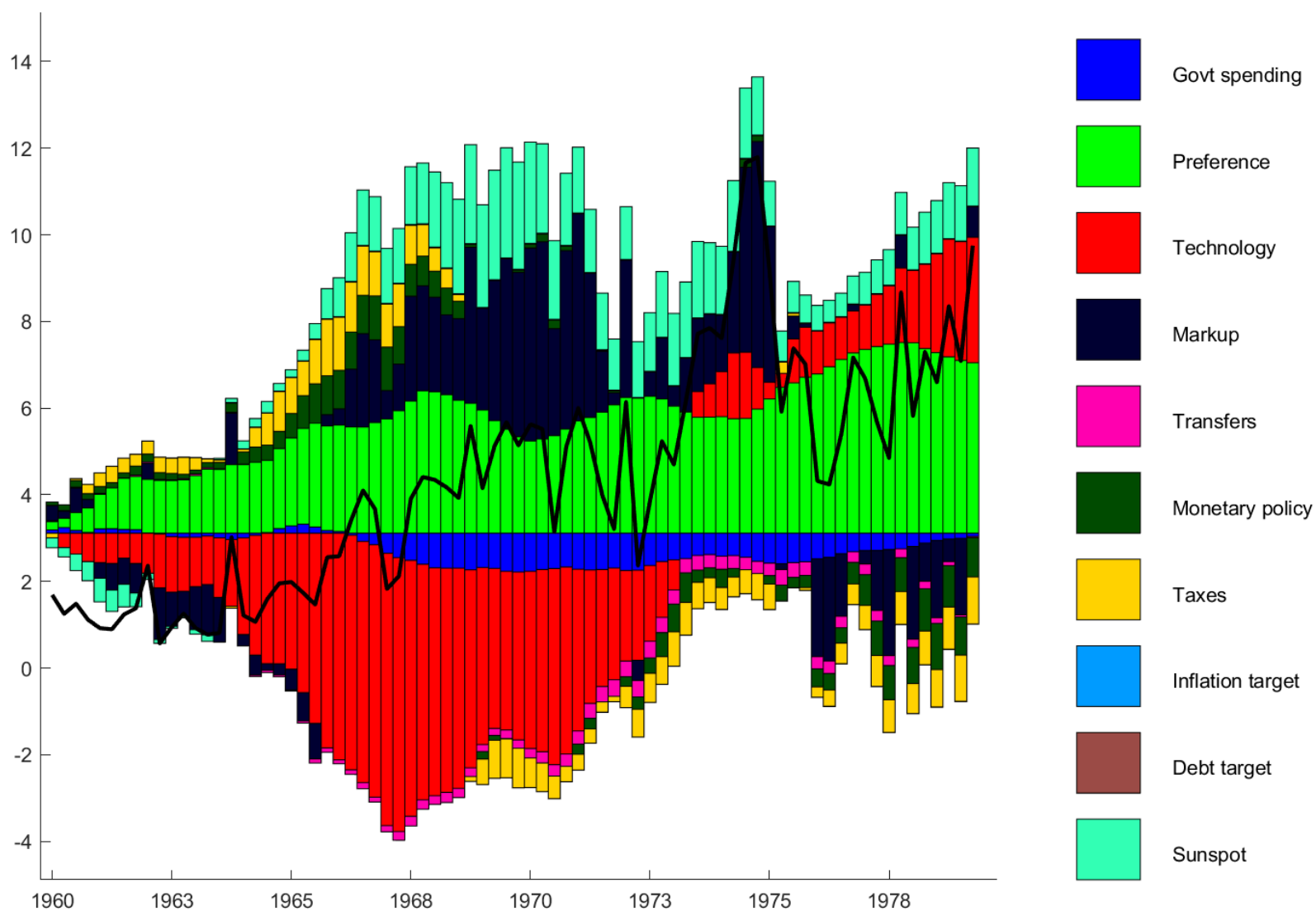

Figure 4: Contribution of each shock to inflation in the indeterminacy regime. The bold black line shows observed inflation. The historical decomposition is conducted at the posterior mean of the indeterminacy regime.

In regime $\mathrm{F}$ the picture looks different. Figure 5 summarizes the findings. Technology and demand shocks played only a minor role in regime F. Instead, the mechanism of the FTPL is clearly present: fiscal actions, government spending in particular, lead to the build-up of inflation. 


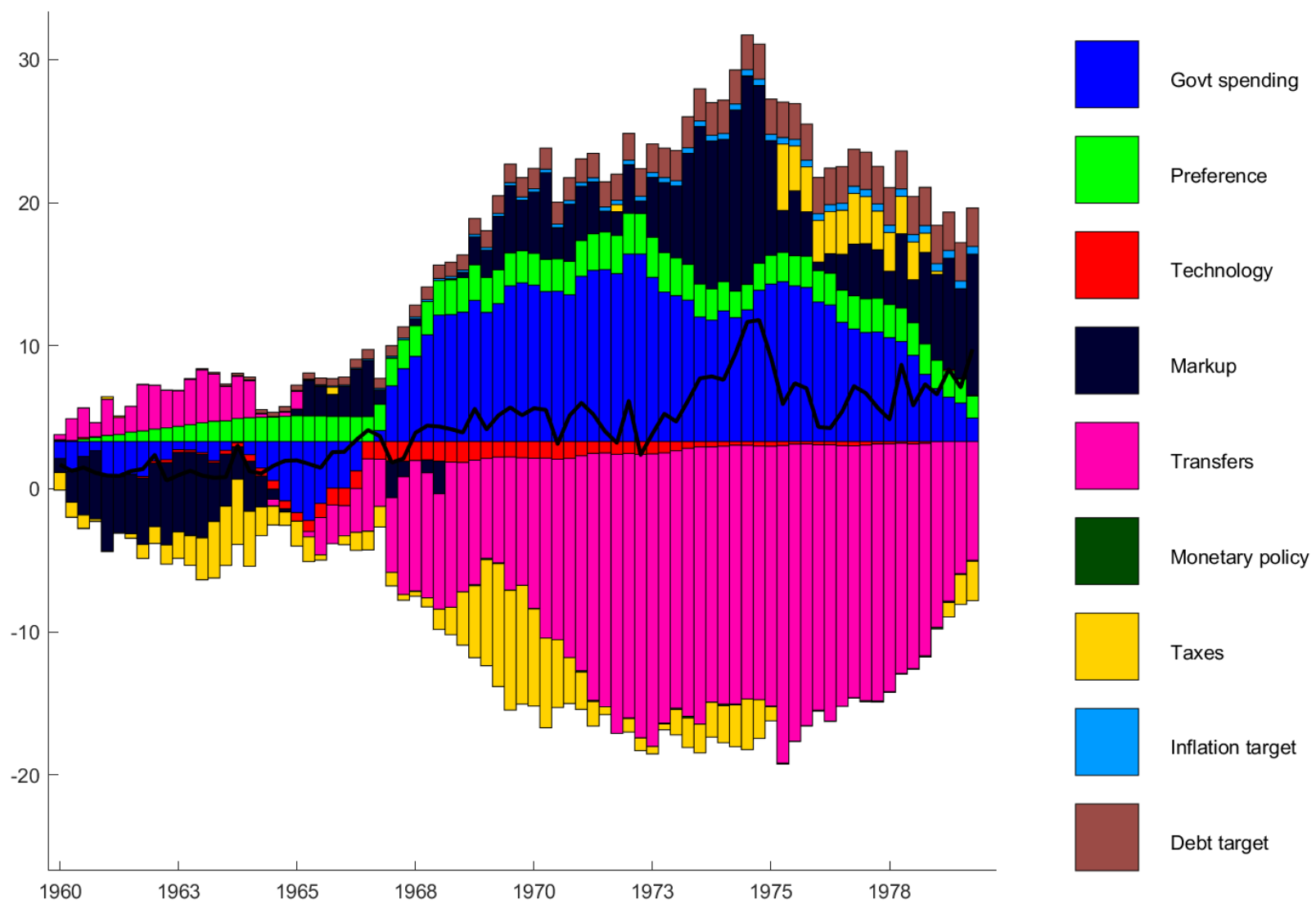

Figure 5: Contribution of each shock to inflation in regime F. The bold black line shows observed inflation. The historical decomposition is conducted at the posterior mean of regime F.

\subsection{Counterfactual analysis}

To further elaborate the role of government spending for pre-Volcker inflation, we carry out a counterfactual analysis. We set the contribution of government spending shocks in each regime to zero and simulate inflation with the remaining shocks. Figure 6 shows the result. In regime $\mathrm{F}$, counterfactual inflation lies considerably below the observed time series. In the indeterminacy regime, on the other hand, the difference between actual and counterfactual inflation is almost negligible.

These results are instructive to evaluate policy measures that effectively brought down pre-Volcker inflation. The Volcker action surely was one possible way to go. By raising interest rates drastically, the central bank credibly signaled that it will take the lead role. 
Reagan complied and backed the monetary policy actions. As a result, the monetary-fiscal policy mix switched to regime M. However, conditional on the results in Figure 6, an alternative policy response crystallizes. Less consumption on the part of the fiscal authority during the 1970s would have also reduced the government spending-to-output ratio and, hence, countered the rising inflation.

Translating the experience of the Great Inflation to the ongoing economic disruption caused by the coronavirus, we learn that monetary and fiscal policy actions have to be determined jointly, independent of a looming deflationary or inflationary period.

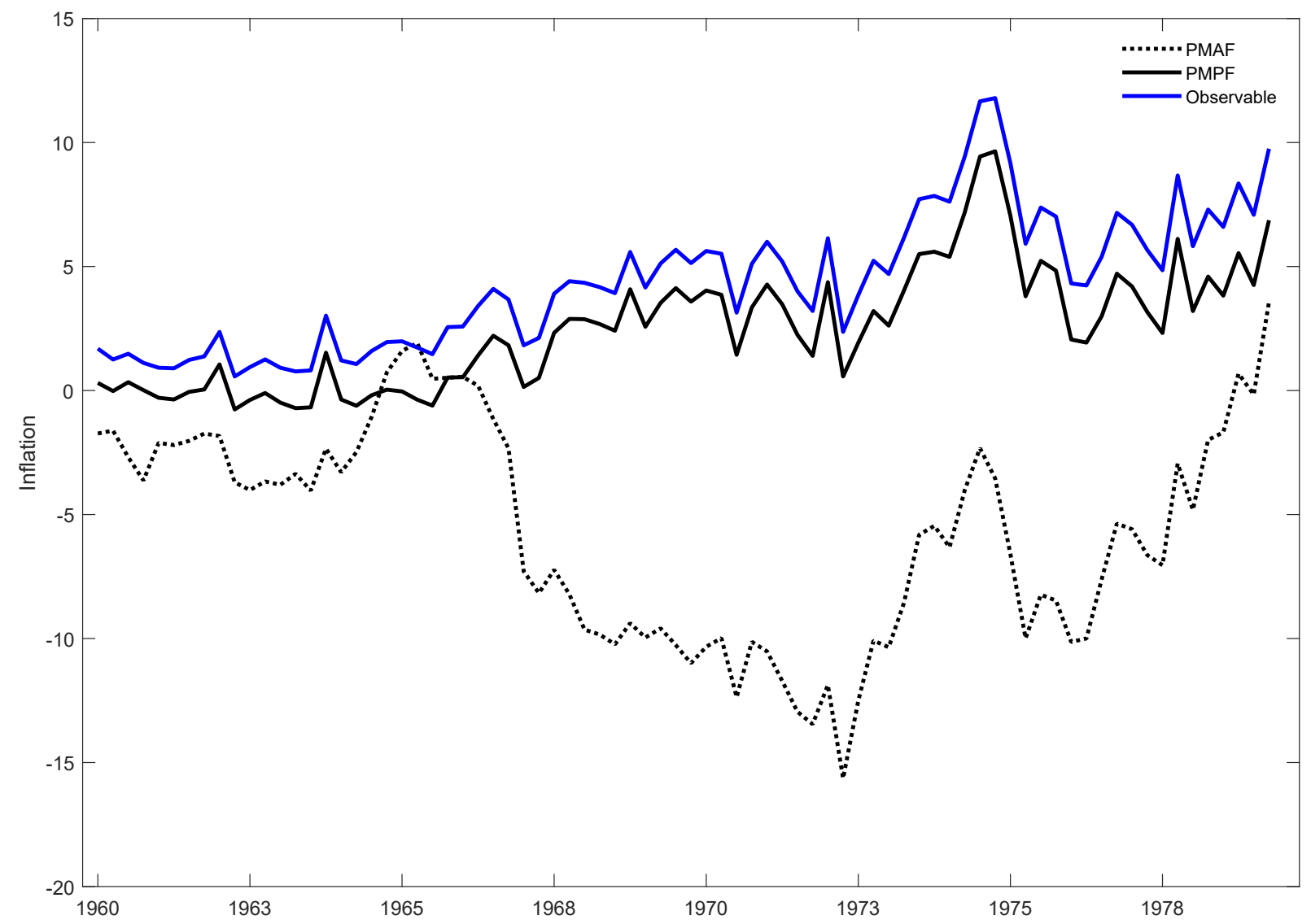

Figure 6: Evolution of inflation (in percentage points) without government spending shock in indeterminacy regime and regime $\mathrm{F}$. The counterfactual analysis is conducted at the posterior mean of each policy regime. 


\section{Conclusion}

Was U.S. fiscal policy in the pre-Volcker period active or passive? We revisit this classic question of modern macroeconomics with a novel empirical toolkit. Using an SMC algorithm, we estimate a DSGE model with monetary-fiscal policy interactions over its entire parameter space. Due to this methodological flexibility, our empirical findings are able to reconcile two opposing strands in the literature: Similar to studies that rely on fixed-regime DSGE models, we find that the indeterminacy regime dominates throughout the 1960:Q1 to 1979:Q2 sample. However, in line with the regime-switching literature, we also find strong evidence for preVolcker periods in which regime $\mathrm{F}$ was in place. Our analysis attributes fiscal policy an

essential role in the build-up of U.S. inflation. Drawing economic lessons for the current COVID-19 pandemic, our analysis suggests that cleverly designed and coordinated active fiscal policy actions could be an effective tool in mitigating the crisis. 


\section{References}

Bhattarai, S., J. W. Lee, And W. Y. Park (2016): "Policy Regimes, Policy Shifts, and U.S. Business Cycles," The Review of Economics and Statistics, 98, 968-983.

Bianchi, F. (2012): "Evolving Monetary/Fiscal Policy Mix in the United States," American Economic Review, 102, 167-72.

Bianchi, F., R. Faccini, And L. Melosi (2020): "Monetary and Fiscal Policies in Times of Large Debt: Unity is Strength," Working Paper 27112, National Bureau of Economic Research.

Bianchi, F. And C. Ilut (2017): "Monetary/Fiscal Policy Mix and Agent's Beliefs," Review of Economic Dynamics, 26, 113-139.

Bognanni, M. And E. Herbst (2018): “A sequential Monte Carlo approach to inference in multiple-equation Markov-switching models," Journal of Applied Econometrics, 33, 126140.

Boivin, J. And M. P. Giannoni (2006): "Has Monetary Policy Become More Effective?" The Review of Economics and Statistics, 88, 445-462.

Chen, X., E. M. Leeper, And C. Leith (2019): "U.S. Monetary and Fiscal Policies Conflict or Cooperation?" Mimeo.

Chib, S. And S. Ramamurthy (2010): "Tailored randomized block MCMC methods with application to DSGE models," Journal of Econometrics, 155, 19-38.

Chopin, N. (2002): "A Sequential Particle Filter Method for Static Models," Biometrika, $89,539-551$.

Clarida, R., J. Gali, and M. Gertler (2000): "Monetary Policy Rules and Macroeconomic Stability: Evidence and Some Theory," The Quarterly Journal of Economics, 115, $147-180$. 
Cochrane, J. H. (2001): "Long-Term Debt and Optimal Policy in the Fiscal Theory of the Price Level," Econometrica, 69, 69-116.

Coibion, O. And Y. Gorodnichenko (2011): "Monetary Policy, Trend Inflation, and the Great Moderation: An Alternative Interpretation," The American Economic Review, $101,341-370$.

Creal, D. (2007): "Sequential Monte Carlo samplers for Bayesian DSGE models," Working paper, Vrije Universitiet Amsterdam.

- (2012): "A Survey of Sequential Monte Carlo Methods for Economics and Finance," Econometric Reviews, 31, 245-296.

Davig, T. And E. M. Leeper (2006): "Fluctuating Macro Policies and the Fiscal Theory," NBER Macroeconomics Annual, 21, 247-298.

Del Moral, P., A. Doucet, And A. Jasra (2006): "Sequential Monte Carlo samplers," Journal of the Royal Statistical Society, B Series, 68, 411-436.

Gelman, A., J. Carlin, H. Stern, D. Dunson, A. Vehtari, and D. Rubin (2013): Bayesian Data Analysis, Third Edition, Chapman \& Hall/CRC Texts in Statistical Science, Taylor \& Francis.

Herbst, E. (2012): "Gradient and hessian-based MCMC for DSGE models," Unpublished manuscript, Federal Reserve Board Washington DC.

Herbst, E. And F. Schorfheide (2014): "Sequential Monte Carlo Sampling for DSGE Models," Journal of Applied Econometrics, 29, 1073-1098.

- (2015): Bayesian Estimation of DSGE Models, Princeton University Press.

LeEPER, E. M. (1991): "Equilibria under 'active' and 'passive' monetary and fiscal policies," Journal of Monetary Economics, 27, 129-147. 
Leeper, E. M., N. Traum, and T. B. Walker (2017): "Clearing Up the Fiscal Multiplier Morass," The American Economic Review, 107, 2409-2454.

Lubik, T. AND F. SChorfheide (2003): "Computing sunspot equilibria in linear rational expectations models," Journal of Economic Dynamics and Control, 28, 273-285.

Lubik, T. A. And F. Schorfheide (2004): "Testing for Indeterminacy: An Application to U.S. Monetary Policy," American Economic Review, 94, 190-217.

Mavroeidis, S. (2010): "Monetary Policy Rules and Macroeconomic Stability: Some New Evidence," American Economic Review, 100, 491-503.

Sims, C. A. (1994): "A Simple Model for Study of the Determination of the Price Level and the Interaction of Monetary and Fiscal Policy," Economic Theory, 4, 381-99.

_ (2002): "Solving Linear Rational Expectations Models," Computational Economics, 20, 1-20.

(2003): "Probability Models For Monetary Policy Decisions," Mimeo.

(2011): "Stepping on a rake: The role of fiscal policy in the inflation of the 1970s," European Economic Review, 55, 48-56.

Woodford, M. (1996): "Control of the Public Debt: A Requirement for Price Stability?" NBER Working Papers 5684, National Bureau of Economic Research. 


\section{Appendix A Model solution}

\section{Appendix A.1 Implementation of the model solution}

The linear rational expectation form of the DSGE model presented in Section 2 is given by

$$
\Gamma_{0}(\vartheta) z_{t}=\Gamma_{1}(\vartheta) z_{t-1}+\Psi(\vartheta) \epsilon_{t}+\Pi(\vartheta) \eta_{t}
$$

$z$ is the vector of state variables, the vector $\epsilon$ includes the exogenous variables, and $\eta$ is a vector of expectation errors. To apply the solution algorithm of Sims (2002), we define, for

a generic variable $\hat{x}_{t}$, the corresponding one-step-ahead rational expectations forecast error as $\eta_{x, t}=\hat{x}_{t}-E_{t-1}\left[\hat{x}_{t}\right]$. In our application, the vectors of the general model form are defined as:

$$
\begin{gathered}
z_{t}=\left[\hat{c}_{t} \hat{\pi}_{t} \hat{a}_{t} \hat{R}_{t} \hat{d}_{t} \hat{Y}_{t} \hat{g}_{t} \hat{u}_{t} \hat{\pi}_{t}^{*} \hat{Y}_{t}^{*} \hat{\tau}_{t} \hat{b}_{t} \hat{b}_{t}^{*} \hat{s}_{t} \hat{g}_{t}^{*} \hat{c}_{t-1} \hat{\pi}_{t-1} \hat{g}_{t-1} \hat{Y}_{t-1}\right]^{\prime} \\
\epsilon_{t}=\left[\epsilon_{g, t} \epsilon_{d, t} \epsilon_{a, t} \epsilon_{u, t} \epsilon_{s, t} \epsilon_{R, t} \epsilon_{\tau, t} \epsilon_{\pi, t} \epsilon_{b, t}\right]^{\prime} \text {, and } \\
\eta_{t}=\left[\eta_{c, t} \eta_{\pi, t}\right]^{\prime}
\end{gathered}
$$

\section{Appendix A.2 Transmission mechanism around the regime bound- aries}

Equation 19 illustrates that indeterminacy changes the nature of the solution in two dimensions. First, the transmission of fundamental shocks $\epsilon_{t}$ is no longer uniquely determined as it additionally depends on the matrix $\tilde{M}$. Second, an exogenous sunspot shock $\zeta_{t}$, unrelated to the fundamental shocks $\epsilon_{t}$, potentially affects the dynamics of the model variables $z_{t}$. Thus, indeterminacy introduces additional parameters.

We denote the standard deviation of the sunspot shock as $\sigma_{\zeta}$ and normalize as Lubik and Schorfheide (2004) $M_{\zeta}$ to unity. Also in accordance with Lubik and Schorfheide (2004), we 
replace $\tilde{M}$ with $\tilde{M}=M^{*}(\vartheta)+M$ to prevent that the transmission of fundamental shocks changes drastically when the boundary between the determinacy regimes and the indeterminacy regime is crossed. Around this boundary, small changes in $\vartheta$ should rather leave the propagation mechanism of structural shocks unaffected. That is why we choose $M^{*}(\vartheta)$ such that the impulse responses $\partial z_{t} / \partial \epsilon_{t}^{\prime}$ become continuous on the boundary. Vector $M$, in contrast, which determines the relationship between fundamental shocks and forecast errors, is estimated. It can be interpreted to capture agents' self-fulfilling beliefs and consists of the following entries: $M=\left[M_{g_{\zeta}}, M_{d_{\zeta}}, M_{a_{\zeta}}, M_{u_{\zeta}}, M_{s_{\zeta}}, M_{R_{\zeta}}, M_{\tau_{\zeta}}, M_{\pi_{\zeta}}, M_{b_{\zeta}}\right]$. For the parameters in $M$, we choose priors centered around zero and, thus, strictly let the data decide how indeterminacy changes the transmission mechanism of structural shocks.

To compute the matrix $M^{*}(\vartheta)$ that guarantees continuous model dynamics on the boundary, we proceed in several steps. First, we construct for every parameter vector $\vartheta \in \Theta^{I}$ (indeterminacy) a reparametrized vector $\vartheta^{*}=g^{*}(\vartheta)$ that lies on the boundary between the indeterminacy and the determinacy regimes. Then, $M^{*}(\vartheta)$ is chosen by a least-squares criterion such that the impulse responses $\frac{\partial z_{t}}{\partial \epsilon_{t}^{\prime}}(\vartheta, M)$ conditional on $\vartheta$ resemble the impulse responses conditional on the vector on the boundary $\frac{\partial z_{t}}{\partial \epsilon_{t}^{\prime}}\left(g^{*}(\vartheta)\right)$. However, the DSGE model, with monetary-fiscal policy interactions presented in subsection 2, gives rise to two different determinate solutions (regime $\mathrm{F}$ and regime $\mathrm{M}$ ) that are generally characterized by different transmission mechanisms. To deal with this ambiguity, we proceed as follows:

1. For every $\vartheta \in \Theta^{I}$ we construct a vector $\vartheta^{M}=g^{M}(\vartheta)$ that demarks the boundary between regime $\mathrm{M}$ and the indeterminacy regime and a vector $\vartheta^{F}=g^{F}(\vartheta)$ that lies on the boundary to regime F. The function $g^{M}(\vartheta)$ is obtained by replacing $\phi_{\pi}$ in the vector $\vartheta$ with

$$
\tilde{\phi}_{\pi}=1-\phi_{Y}\left(\frac{1-\tilde{\beta}}{\tilde{\kappa}}\right)
$$


The function $g^{F}(\vartheta)$ is obtained by replacing $\psi_{b}$ in the vector $\vartheta$ with

$$
\tilde{\psi}_{b}=\frac{1}{\beta}-1
$$

2. We solve the model successively with the reparametrized vectors $\vartheta^{M}$ and $\vartheta^{F}$ and compute

$$
\begin{gathered}
M^{M}(\vartheta)=\left[\Gamma_{0, \zeta}^{M}(\vartheta)^{\prime} \Gamma_{0, \zeta}^{M}(\vartheta)\right]^{-1} \Gamma_{0, \zeta}^{M}(\vartheta)^{\prime}\left[\Gamma_{0, \epsilon}^{M}\left(g^{M}(\vartheta)\right)-\Gamma_{0, \epsilon}^{M}(\vartheta)\right], \text { and } \\
M^{F}(\vartheta)=\left[\Gamma_{0, \zeta}^{F}(\vartheta)^{\prime} \Gamma_{0, \zeta}^{F}(\vartheta)\right]^{-1} \Gamma_{0, \zeta}^{F}(\vartheta)^{\prime}\left[\Gamma_{0, \epsilon}^{F}\left(g^{F}(\vartheta)\right)-\Gamma_{0, \epsilon}^{F}(\vartheta)\right] .
\end{gathered}
$$

3. To choose the $M^{*}(\vartheta)$ that minimizes the discrepancy between $\frac{\partial z_{t}}{\partial \epsilon_{t}^{\prime}}(\vartheta, M)$ and $\frac{\partial z_{t}}{\partial \epsilon_{t}^{\prime}}\left(g^{*}(\vartheta)\right)$, we compute the distances to the respective boundaries as

$$
\begin{gathered}
D^{M}=\left[\Gamma_{0, \epsilon}^{M}\left(g^{M}(\vartheta)\right)-\Gamma_{0, \epsilon}^{M}(\vartheta)\right]-\Gamma_{0, \zeta}^{M}(\vartheta) M^{M}(\vartheta), \text { and } \\
D^{F}=\left[\Gamma_{0, \epsilon}^{F}\left(g^{F}(\vartheta)\right)-\Gamma_{0, \epsilon}^{F}(\vartheta)\right]-\Gamma_{0, \zeta}^{F}(\vartheta) M^{F}(\vartheta)
\end{gathered}
$$

4. As, in our model, all fundamental shocks are assumed to be independent from each other, we compute the Euclidean norm of each column in $D^{*}$, sum them up, and, finally, choose the $M^{*}(\vartheta)$ that corresponds with ${ }^{17}$

$$
\min \left[\sum_{j=1}^{9}\left\|d_{j}^{M}\right\|_{2}, \sum_{j=1}^{9}\left\|d_{j}^{F}\right\|_{2}\right]
$$

Here, we show plots to demonstrate that our approach delivers effectively continuous impulse response functions on the boundary between policy regimes. We draw 20,000 times from the prior distribution and solve with each draw the model. If a draw lies in the

\footnotetext{
${ }^{17}$ For matrix $D^{*}=\left(d_{i j}^{*}\right)$, its i-th row and $\mathrm{j}$-th column are denoted by $d_{i}^{*}$ and $d_{j}^{*}$, respectively.
} 
indeterminacy region, we first determine with the least-square criterion if it is closer to the monetary (regime M) or the fiscal boundary (regime F) of the determinacy region. Then we conduct the following steps:

If the draw's position in the parameter space is closer to the monetary boundary, we reparametrize the parameter vector to lie on the monetary boundary.

1. We solve the model on the boundary and compute impulse responses.

2. We step numerically from the boundary into the indeterminacy region, solve the model and compute impulse responses.

3. To check if the transmission mechanism changes when crossing the boundary, we compute the difference between the impulse responses on the boundary, and the impulse responses from the indeterminacy region.

We repeat the three steps for the draws that are located closer to the fiscal boundary. Figures 7 and 8 show that the impulse responses are nearly congruent. 

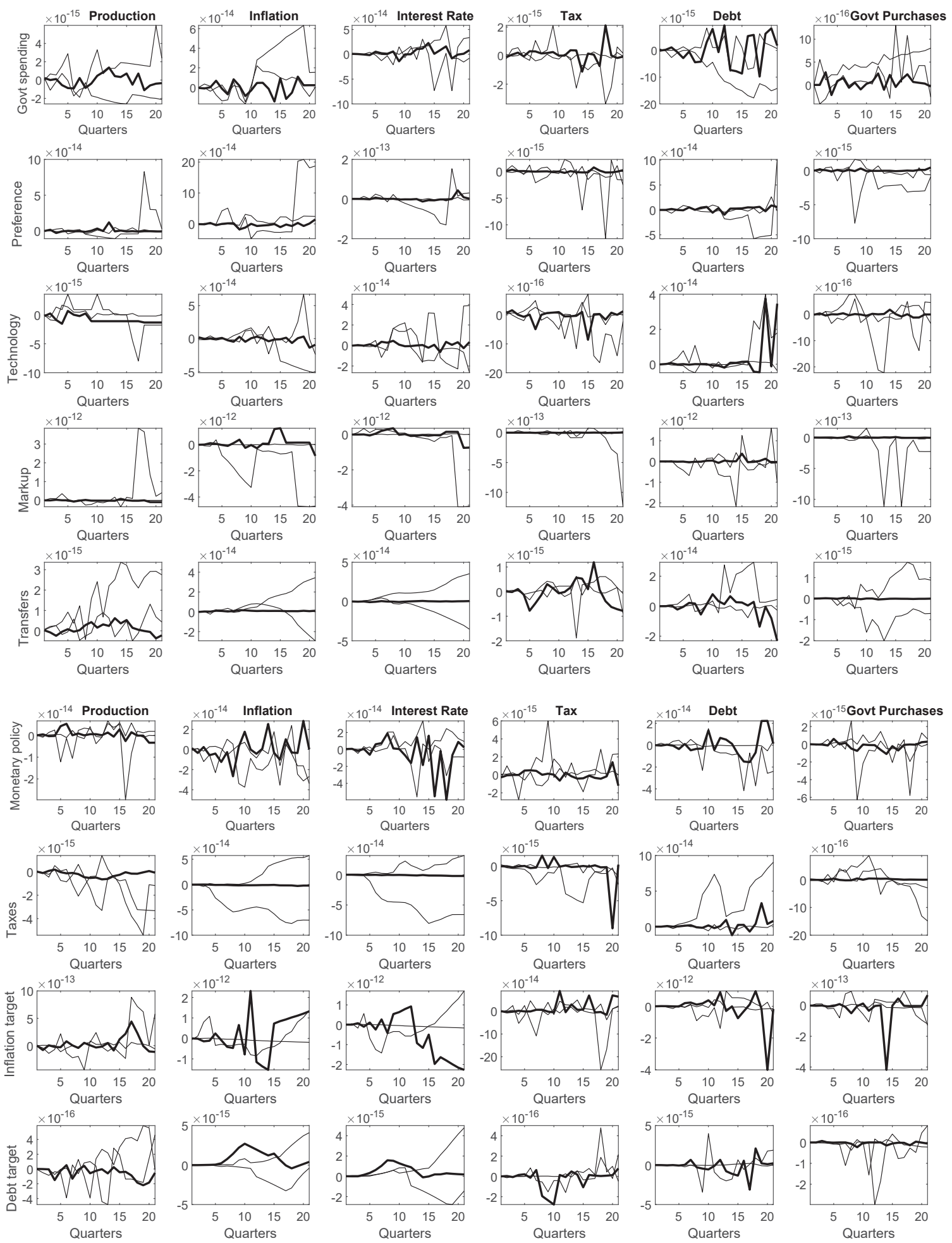

Figure 7: Difference of IRFs computed in the determinacy and the indeterminacy region around the monetary boundary. The bold line shows posterior means and the solid line $90 \%$ credible sets. 

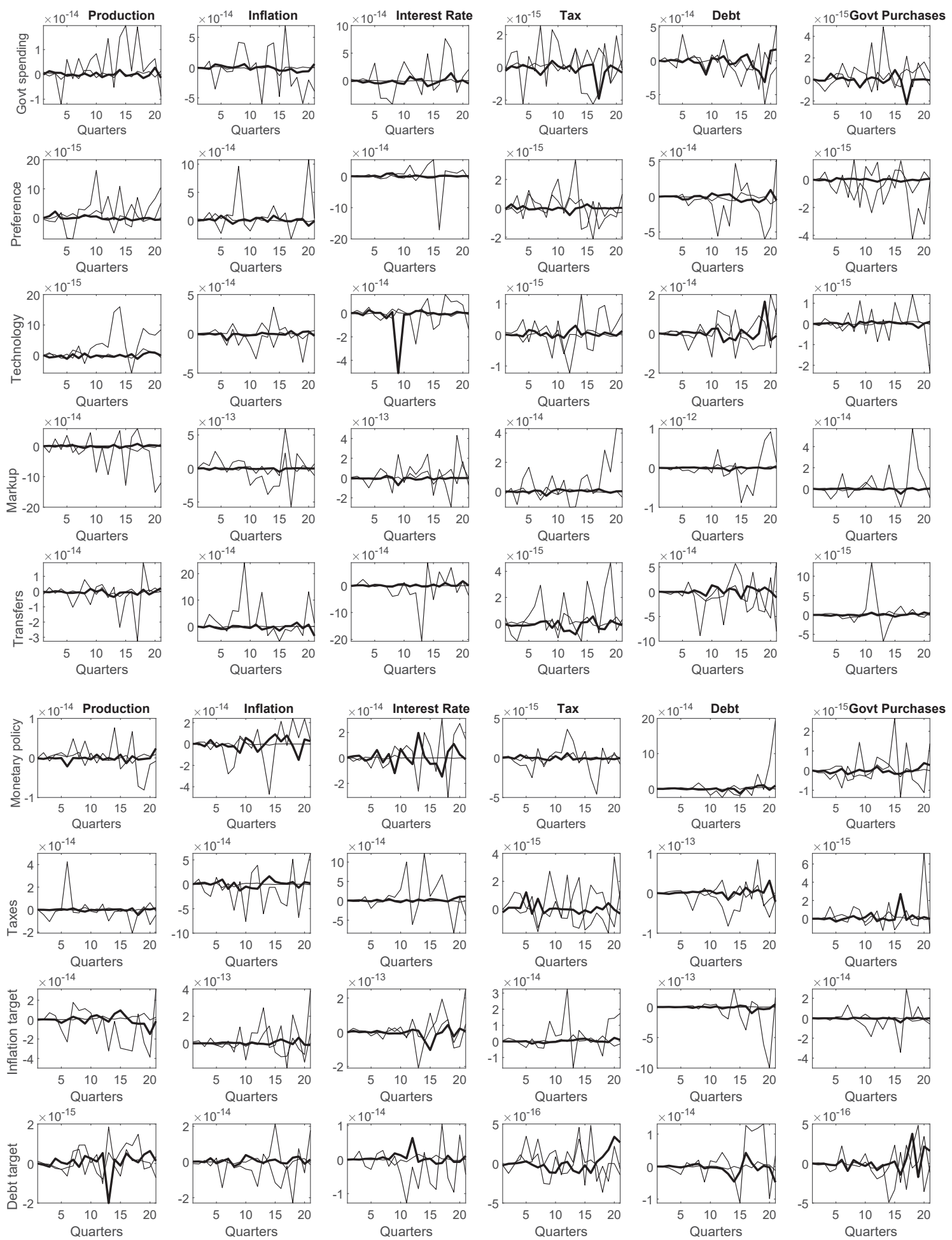

Figure 8: Difference of IRFs computed in the determinacy and the indeterminacy region around the fiscal boundary. The bold line shows posterior means and the solid line $90 \%$ credible sets. 


\section{Appendix B Prior implications}

Here, we show results of a prior predictive analysis for the prior specification outlined in Section 3.1. In particular, we take 20,000 draws from the prior and simulate with these draws 20,000 times the model's observables.
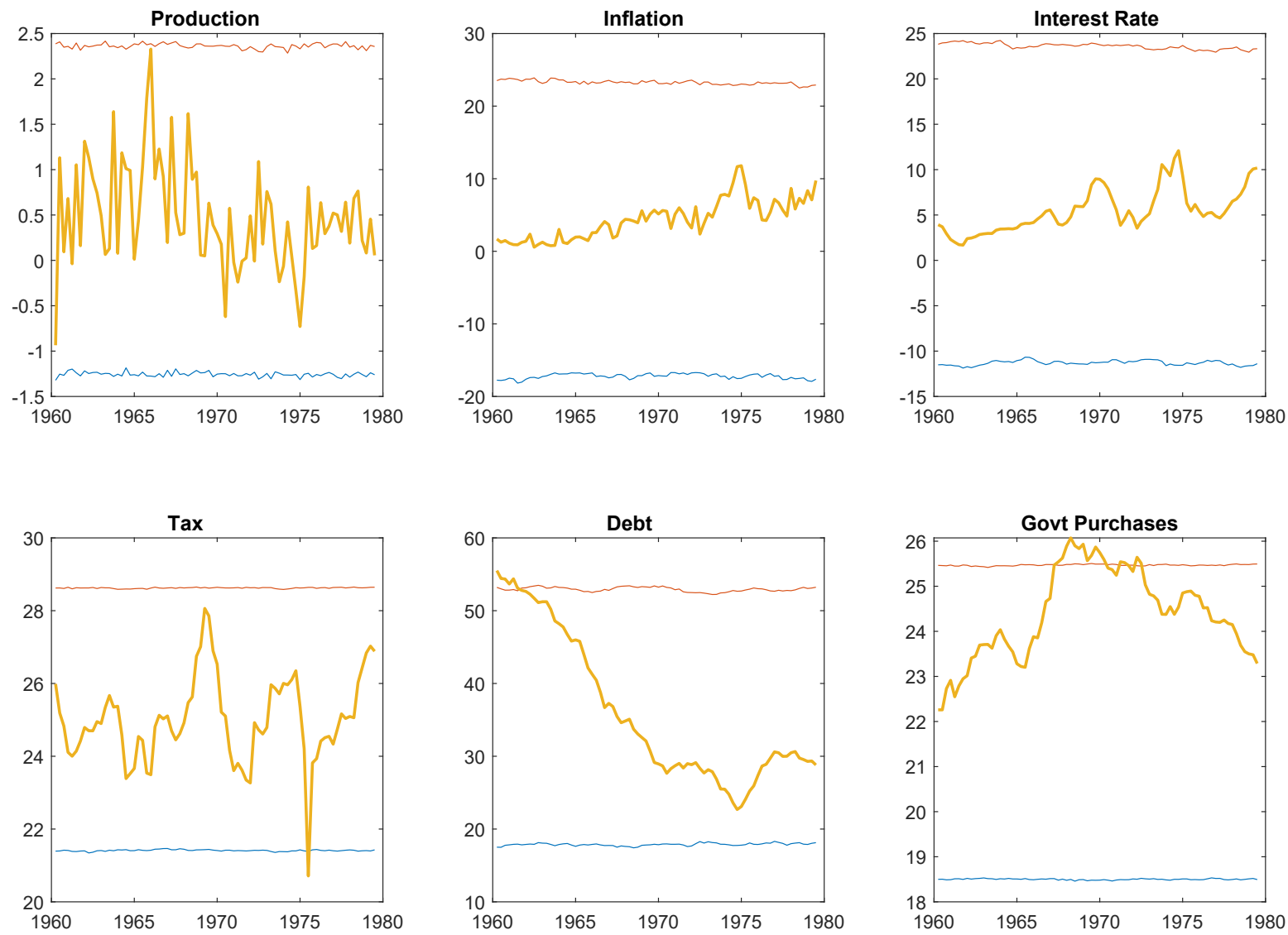

Figure 9: Simulated model observables vs. real data for 1960:Q1 to 1979:Q2. The bold yellow line shows the actual time series we use for estimating the model. The blue and the red line show the $90 \%$ intervall of the simulated time series. 


\section{Appendix C Data description}

We use the dataset of Bhattarai et al. (2016). Unless otherwise noted, the data is retrieved from the National Income and Product Accounts Tables published by the Bureau of Economic Analysis. All time series in nominal values are converted to real values by dividing them by the GDP deflator.

Per capita output: Per capita output is the sum of personal consumption of nondurables and services, and government consumption divided by civilian noninstitutional population. Civilian noninstitutional poulation is taken from the FRED database of the Federal Reserve Bank of St. Louis.

Inflation: The gross inflation rate is the annualized GDP deflator.

Interest rate: The annualized nominal interest rate is the effective federal funds rate from the FRED database of the Federal Reserve Bank of St. Louis.

Tax revenues: The tax-revenues-to-output ratio is defined as the sum of current tax receipts and contributions for government social insurance divided by output.

Government debt: Government debt corresponds to the market value of privately held gross federal debt, retrieved from the Federal Reserve Bank of Dallas. The government debt-to-output ratio is obtained by dividing the series by output.

Government spending: The government spending-to-output ratio is defined as government consumption divided by output. 
The relationship between observables and model variables is given by

$\left[\begin{array}{c}100 \times \Delta \ln \text { Production }_{t} \\ \text { Inflation }_{t}(\%) \\ \text { Interest }_{t}(\%) \\ \operatorname{TaxRev}_{t}(\%) \\ \text { GovtDebt }_{t}(\%) \\ \operatorname{GovtPurch}_{t}(\%)\end{array}\right]=\left[\begin{array}{c}a \\ 4 \pi \\ 4(a+\pi+\mu) \\ \tau \\ b \\ g\end{array}\right]+\left[\begin{array}{c}\hat{Y}_{t}-\hat{Y}_{t-1}+\hat{a}_{t} \\ 4 \hat{\pi}_{t} \\ 4 \hat{R}_{t} \\ \hat{\tau}_{t} \\ \hat{b}_{t} \\ \hat{g}_{t}\end{array}\right]$




\section{Appendix D SMC algorithm}

This appendix completes the technical description of the implemented SMC algorithm. In terms of exposition and notation it draws heavily on Herbst and Schorfheide $(2014,2015)$ and Bognanni and Herbst (2018).

\section{Appendix D.1 SMC with likelihood tempering}

1. The SMC is initialized by drawing the particles of the first stage $\left(n=1 ; \delta_{1}=0\right)$ from the prior density. ${ }^{18}$

$$
\vartheta_{1}^{i} \stackrel{i . i . d .}{\sim} p(\vartheta) \quad i=1, \ldots, N
$$

In the first stage, each particle receives equal weight such that $W_{1}^{i}=1$.

\section{Recursions:}

for $\mathrm{n}=2: N_{\delta}$

1. Correction: Reweight the particles from stage $n-1$ by defining the incremental and normalized weights as

$$
\tilde{w}_{n}^{i}=\left[p\left(Y \mid \vartheta_{n-1}^{i}\right)\right]^{\delta_{n}-\delta_{n-1}}, \quad \tilde{W}_{n}^{i}=\frac{\tilde{w}_{n}^{i} W_{n-1}^{i}}{\frac{1}{N} \sum_{i=1}^{N} \tilde{w}_{n}^{i} W_{n-1}^{i}}, i=1, \ldots, N
$$

2. Selection: Check particle degeneracy by computing the effective sample size

$$
E S S_{n}=\frac{N}{\frac{1}{N} \sum_{i=1}^{N}\left(\tilde{W}_{n}^{i}\right)^{2}}
$$

The ESS monitors the variance of the particle weights. The larger this variance, the more inefficient runs the sampler. If the distribution of particle weights becomes too uneven, resampling the particles helps to improve accuracy.

if $E S S_{n}<N / 2$

\footnotetext{
${ }^{18}$ To ease notation in Appendix D, we assume that the parameters in $M$ are part of $\vartheta$.
} 
Resample the particles via systematic resampling and set the weights to uniform

$$
W_{n}^{i}=1, \quad \hat{\vartheta}_{n}^{i} \sim\left\{\vartheta_{n-1}^{j}, \tilde{W}_{n}^{j}\right\}_{j=1, \ldots, N} \quad i=1, \ldots, N
$$

else

$$
W_{n}^{i}=\tilde{W}_{n}^{i}, \quad \hat{\vartheta}_{n}^{i}=\vartheta_{n-1}^{i}, \quad i=1, \ldots, N
$$

end if

3. Mutation: Propagate each particle $\left\{\tilde{\vartheta}_{N}^{i}, W_{n}^{i}\right\}$ via $M_{M H}$ steps of a RWMH with $N_{\text {blocks }}$ random blocks. See Appendix D.2 for further details.

end for

\section{Process posterior draws.}

\section{Appendix D.2 Mutation step}

In this section, we specify the RWMH sampler we use for particle mutation. In accordance with Herbst and Schorfheide (2014) and Bognanni and Herbst (2018) the RWMH steps in our application are characterized by two features. First, we reduce the dimensionality of the parameter vector $\vartheta$ by spliting it into $N_{\text {blocks }}$ blocks, thus making it easier to approximate the target density in each of the RWMH's $M_{M H}$ steps. ${ }^{19}$ Second, we scale the variance of the proposal density adaptively. Let $\hat{\Sigma}_{n}$ be the estimate of the covariance of $p_{n}(\vartheta \mid Y)$ after the selection step and $c_{n}$ be a scaling factor. We set $c_{n}$ as a function of the previous stage's scaling factor $c_{n-1}$ and the average empirical acceptance rate of the previous stage's mutation step $\hat{A}_{n-1}$. We target an acceptance rate of $25 \%$ and, hence, increase $c_{n}$ if the acceptance rate in stage $n-1$ was too high or decrease $c_{n}$ if it was too low. In particular, the functional form is given by $\hat{c}_{n}=\hat{c}_{n-1} f\left(\hat{A}_{n-1}\right)$, where $f(x)=0.95+0.1 \frac{e^{16(x-0.25)}}{1+e^{16(x-0.25)}}$.

\footnotetext{
${ }^{19}$ Chib and Ramamurthy (2010) and Herbst (2012) provide evidence that parameter blocking is benefical for estimating DSGE models.
} 
1. In every $n$ stage after the selection step, create a random partitioning of the parameter vector $\vartheta$ into $N_{\text {blocks }}$. $b$ denotes the block of the parameter vector such that $\vartheta_{b, n}^{i}$ refers to the $b$ elements of the $i$ th particle, and $\vartheta_{<b, n}^{i}$ denotes the remaining partitions.

2. Compute an estimate of the covariance of the parameters as

$$
\hat{\Sigma}_{n}=\sum_{i=1}^{N} W_{n}^{i}\left(\hat{\vartheta}_{n}^{i}-\hat{\mu}_{n}\right)\left(\hat{\vartheta}_{n}^{i}-\hat{\mu}_{n}\right)^{\prime} \quad \text { with } \quad \hat{\mu}_{n}=\sum_{i=1}^{N} W_{n}^{i} \hat{\vartheta}_{n}^{i}
$$

The covariance for the $b$ th block is given by

$$
\hat{\Sigma}_{b, n}=\left[\hat{\Sigma}_{n}\right]_{b, b}-\left[\hat{\Sigma}_{n}\right]_{b,-b}\left[\hat{\Sigma}_{n}\right]_{-b,-b}^{-1}\left[\hat{\Sigma}_{n}\right]_{-b, b},
$$

where $\left[\hat{\Sigma}_{n}\right]_{b, b}$ refers to the $b$ th block of $\hat{\Sigma}_{n}$.

\section{MH steps:}

for $\mathrm{m}=1: M_{M H}$

for $\mathrm{b}=1: N_{\text {blocks }}$

1. Draw a proposal density $\vartheta_{b}^{*} \sim N\left(\vartheta_{m-1, b, n}^{i}, c_{n}^{2} \hat{\Sigma}_{b, n}\right)$.

$\vartheta^{*}=\left[\vartheta_{m,<b, n}^{i}, \vartheta_{b}^{*}, \vartheta_{m-1,>b, n}^{i}\right]$ and $\vartheta_{m, n}^{i}=\left[\vartheta_{m,<b, n}^{i}, \vartheta_{m-1, \geq b, n}^{i}\right]$.

2. With probability

$$
\alpha=\min \left\{\frac{\left[p\left(Y \mid \vartheta^{*}\right)\right]^{\delta_{n}} p\left(\vartheta^{*}\right)}{\left[p\left(Y \mid \vartheta_{m, n}^{i}\right)\right]^{\delta_{n}} p\left(\vartheta_{m, n}^{i}\right)}, 1\right\}
$$

set $\vartheta_{m, b, n}^{i}=\vartheta_{b}^{*}$. Otherwise, set $\vartheta_{m, b, n}^{i}=\vartheta_{m-1, b, n}^{i}$.

end for

end for 


\section{Appendix E Prior specification of Bhattarai et al. (2016)}

Here, we show results of a prior predictive analysis for the prior specification employed in Bhattarai et al. (2016). To ensure that we completely impose a particular policy regime during estimation, we follow the authors and reparameterize the model by introducing new parameters $\phi_{\pi}^{*}$ and $\psi_{b}^{*}$, which measure the distance from the boundary of active and passive policies. The boundary for monetary policy and fiscal policy is denoted by $\tilde{\phi}_{\pi}$ and $\tilde{\psi}_{b}$, respectively (see Equation 23 and 24 in Appendix A.2.). Then, set

$$
\begin{aligned}
\phi_{\pi}=\tilde{\phi}_{\pi}+\phi_{\pi}^{*} ; & \psi_{b}=\tilde{\psi}_{b}+\psi_{b}^{*} \\
\phi_{\pi}=\tilde{\phi}_{\pi}-\phi_{\pi}^{*} ; & \psi_{b}=\tilde{\psi}_{b}-\psi_{b}^{*} \\
\phi_{\pi}=\tilde{\phi}_{\pi}-\phi_{\pi}^{*} ; & \psi_{b}=\tilde{\psi}_{b}+\psi_{b}^{*}
\end{aligned}
$$

for regime $\mathrm{M}$, regime $\mathrm{F}$, and the indeterminacy regime, respectively. $\phi_{\pi}^{*}$ and $\psi_{b}^{*}$ are assumed to have a Gamma prior distribution. $\phi_{\pi}^{*}$ has a mean of 0.5 and a standard deviation of 0.2. $\psi_{b}^{*}$ has a mean of 0.05 and a standard deviation of 0.04. Bhattarai et al. (2016) estimate

for each regime $\phi_{\pi}^{*}$ and $\psi_{b}^{*}$ and compute the implied value for $\phi_{\pi}$ and $\psi_{b}$, respectively. We follow them in this approach. More details can be found in the original study.

For each policy regime we take 20,000 draws from the prior and simulate with these draws 20,000 times the model's observables. As can be seen in Figures 10 to 12, the prior choice of Bhattarai et al. (2016) leads to simulated model observables with a very broad range. As we initialize the SMC algorithm from the prior, we carefully tailored a prior that results in more realistic model implications, but nevertheless remains agnostic about the prevailing policy regime (see Section 3.1 and Appendix B.). 

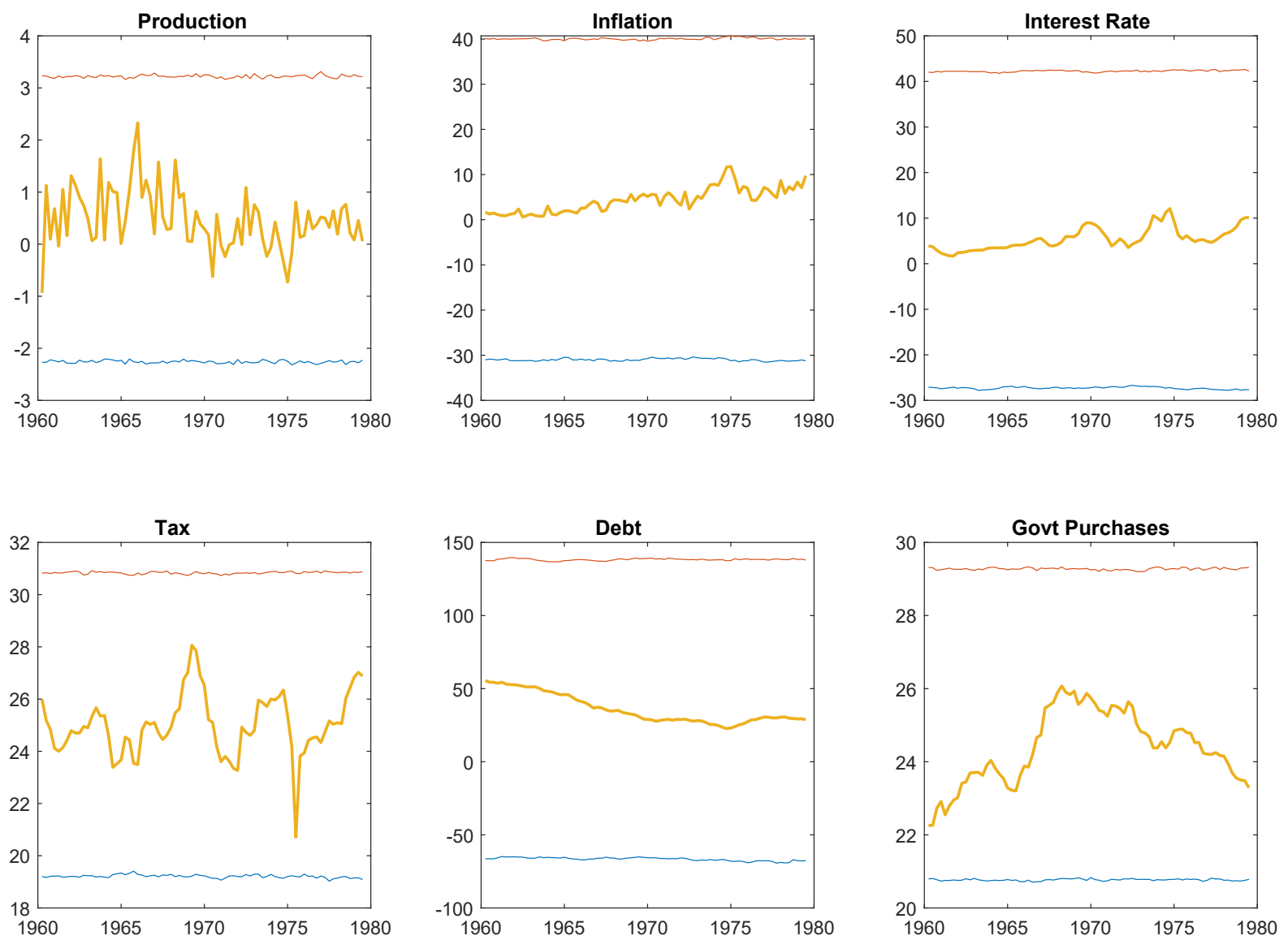

Figure 10: Simulated model observables vs. real data for 1960:Q1 to 1979:Q2 for regime M. The bold yellow line shows the actual time series we use for estimating the model. The blue and the red lines show the $90 \%$ intervall of the simulated time series. 

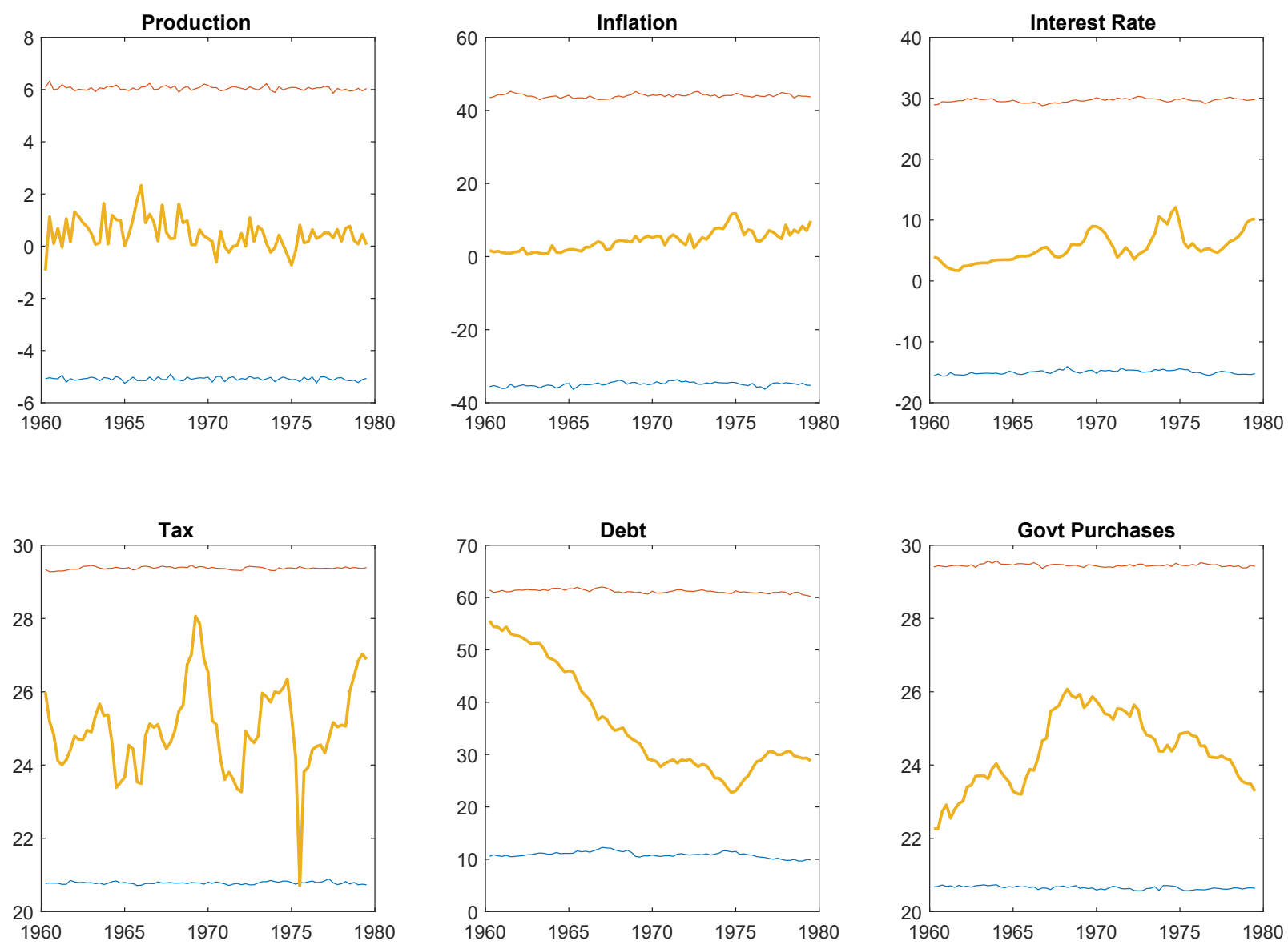

Figure 11: Simulated model observables vs. real data for 1960:Q1 to 1979:Q2 for regime F. The bold yellow line shows the actual time series we use for estimating the model. The blue and the red lines show the $90 \%$ intervall of the simulated time series. 

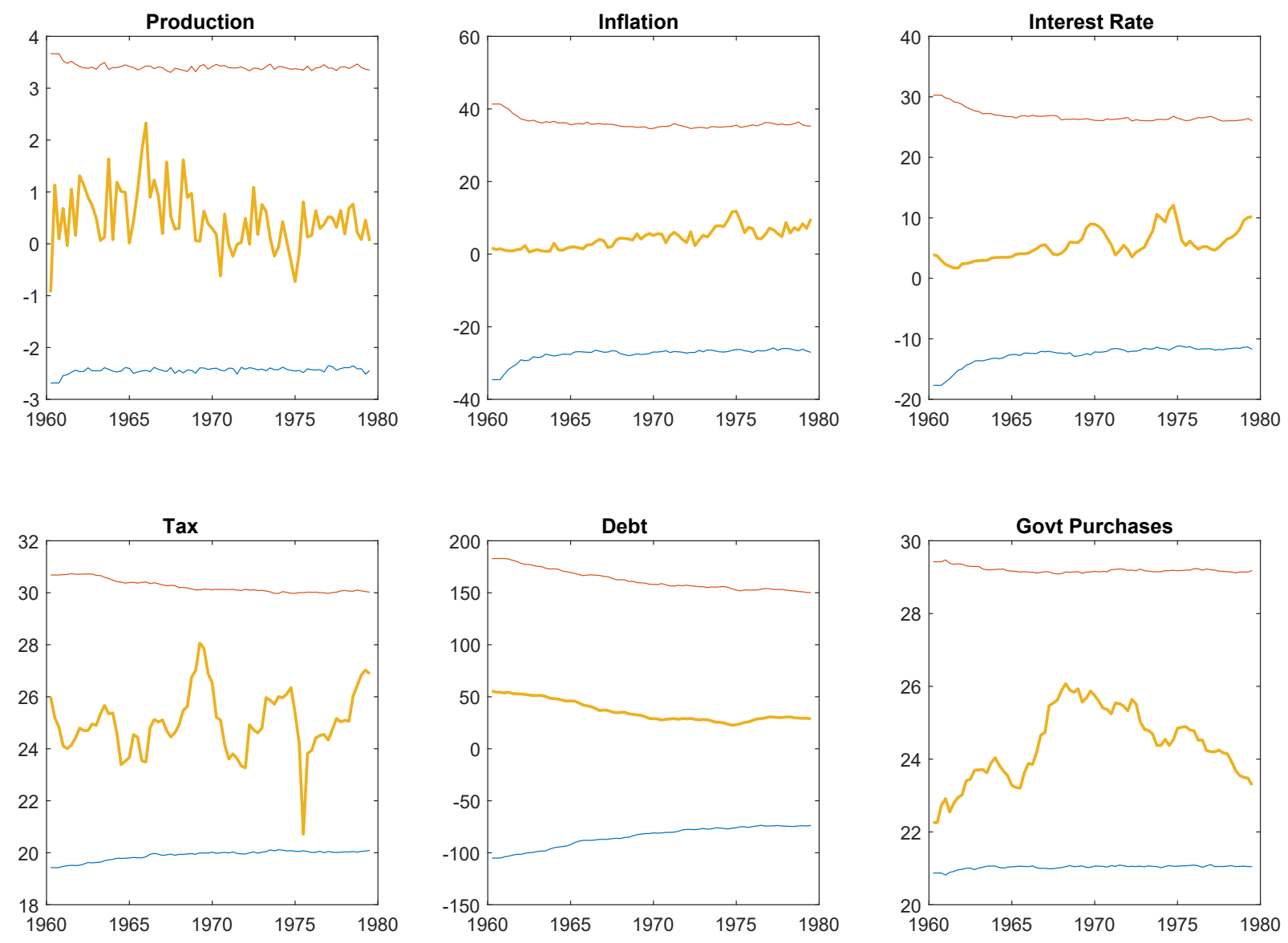

Figure 12: Simulated model observables vs. real data for 1960:Q1 to 1979:Q2 for the indeterminacy regime. The bold yellow line shows the actual time series we use for estimating the model. The blue and the red lines show the $90 \%$ intervall of the simulated time series. 


\section{Appendix F Posterior estimates}

\section{Appendix F.1 Restricted estimation}

Here, we show plots of the prior and posterior densities for the remaining parameters from the restricted estimation with the SMC sampler and tables that summarize the estimation results for regime $\mathrm{F}$ and the indeterminacy regime. In a first step, we show the results of the restricted estimation with the prior specification, as in Bhattarai et al. (2016). In a second step, we show the results of the restricted estimation, with our prior specification and the reparametrized policy parameters $\phi_{\pi}^{*}$ and $\psi_{b}^{*}$ as in Bhattarai et al. (2016).

Restricted estimation - prior as in Bhattarai et al. (2016)
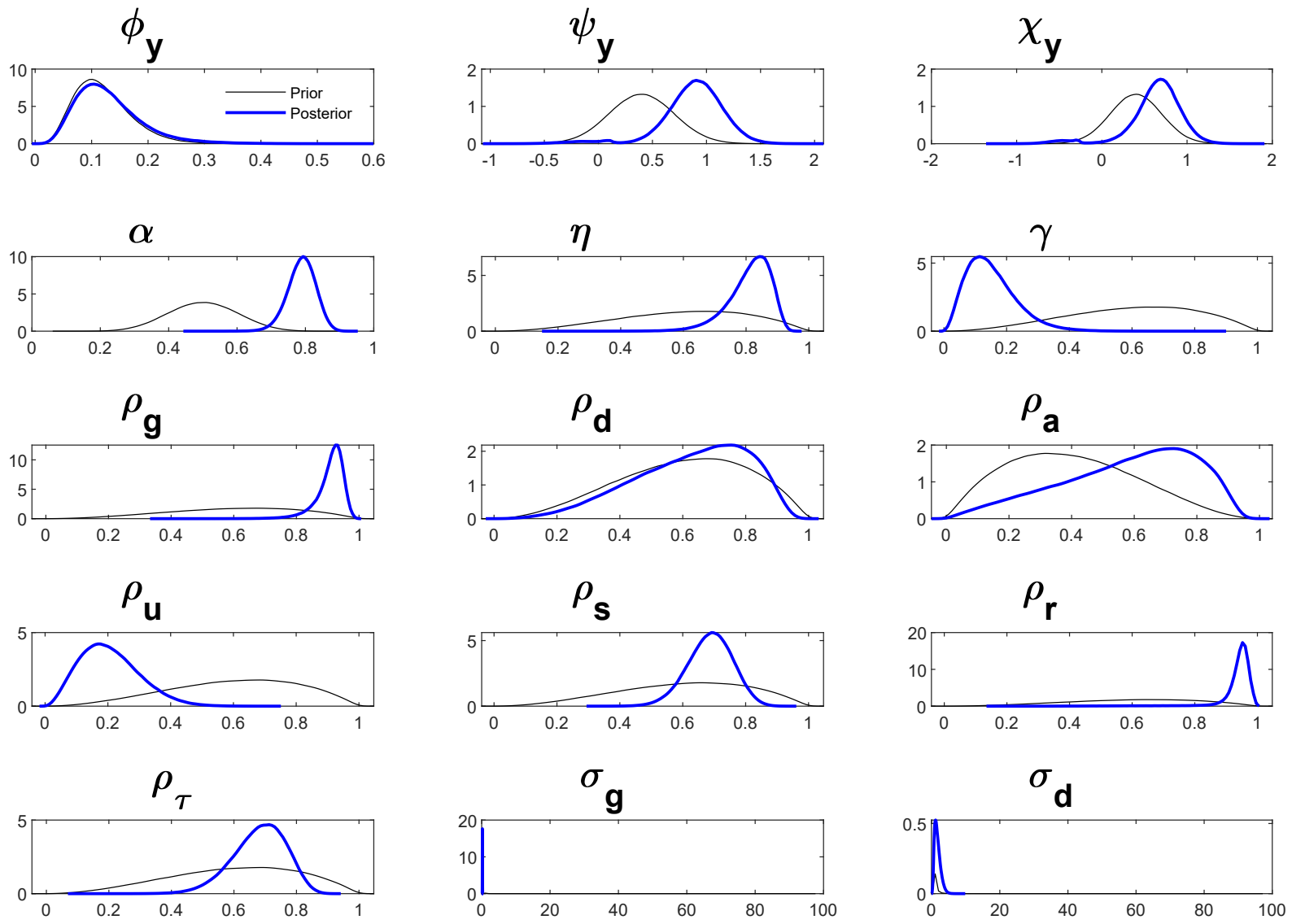

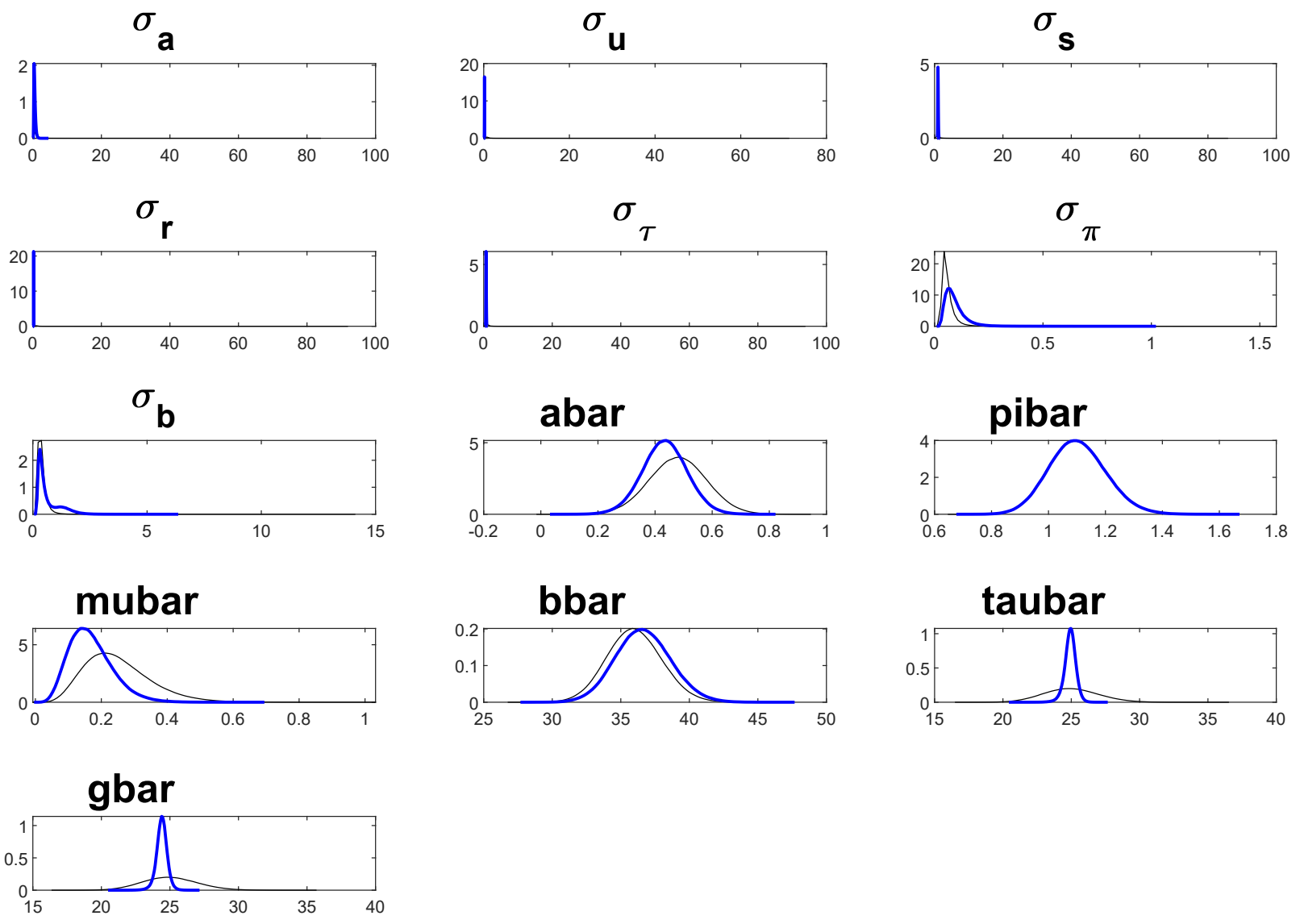

Figure 13: Prior and posterior densities of the estimated model parameters for regime F. The blue bold line depicts the posterior density, the black line the prior density. The prior densities are specified as in Bhattarai et al. (2016). 
Table 6: Posterior distributions for estimated parameters (Regime F)

\begin{tabular}{|c|c|c|c|}
\hline \multirow{2}{*}{ Parameter } & \multicolumn{3}{|c|}{ Posterior } \\
\hline & Mean & SD & 90 percent credible set \\
\hline \multicolumn{4}{|l|}{ Monetary policy } \\
\hline$\phi_{\pi}$, interest rate response to inflation & 0.71 & 0.13 & {$[0.53,0.9]$} \\
\hline$\phi_{\pi}^{*}$, distance to monetary boundary & 0.27 & 0.13 & {$[0.09,0.46]$} \\
\hline$\phi_{Y}$, interest rate response to output & 0.13 & 0.06 & {$[0.04,0.21]$} \\
\hline$\rho_{R}$, response to lagged interest rate & 0.93 & 0.07 & {$[0.9,0.99]$} \\
\hline \multicolumn{4}{|l|}{ Fiscal policy } \\
\hline$\psi_{b}$, tax response to lagged debt & -0.08 & 0.04 & {$[-0.14,-0.02]$} \\
\hline$\psi_{b}^{*}$, distance to fiscal boundary & 0.08 & 0.04 & {$[0.02,0.14]$} \\
\hline$\psi_{Y}$, tax response to output & 0.87 & 0.3 & {$[0.49,1.33]$} \\
\hline $\begin{array}{l}\chi_{Y} \text {, govt spending response to } \\
\text { lagged output }\end{array}$ & 0.63 & 0.31 & {$[0.24,1.11]$} \\
\hline$\rho_{g}$, response to lagged govt spending & 0.91 & 0.04 & {$[0.85,0.97]$} \\
\hline$\rho_{\tau}$, response to lagged taxes & 0.68 & 0.08 & {$[0.55,0.82]$} \\
\hline \multicolumn{4}{|l|}{ Preference and $\mathrm{HHs}$} \\
\hline$\eta$, habit formation & 0.81 & 0.07 & {$[0.71,0.91]$} \\
\hline$\mu:=100\left(\beta^{-1}-1\right)$, discount factor & 0.17 & 0.07 & {$[0.06,0.27]$} \\
\hline \multicolumn{4}{|l|}{ Frictions } \\
\hline$\alpha$, price stickiness & 0.79 & 0.04 & {$[0.72,0.86]$} \\
\hline$\gamma$, price indexation & 0.15 & 0.08 & {$[0.03,0.27]$} \\
\hline \multicolumn{4}{|l|}{ Shocks } \\
\hline$\rho_{d}$, preference & 0.63 & 0.18 & {$[0.35,0.91]$} \\
\hline$\rho_{a}$, technology & 0.58 & 0.21 & {$[0.24,0.9]$} \\
\hline$\rho_{u}$, cost-push & 0.21 & 0.09 & {$[0.05,0.35]$} \\
\hline$\rho_{s}$, transfers & 0.69 & 0.07 & {$[0.57,0.8]$} \\
\hline$\sigma_{g}$, govt spending & 0.21 & 0.02 & {$[0.18,0.25]$} \\
\hline$\sigma_{d}$, preference & 1.71 & 0.89 & {$[0.41,3.03]$} \\
\hline$\sigma_{a}$, technology & 0.54 & 0.25 & {$[0.19,0.89]$} \\
\hline$\sigma_{u}$, cost-push & 0.18 & 0.02 & {$[0.14,0.22]$} \\
\hline$\sigma_{s}$, transfers & 1.01 & 0.09 & {$[0.87,1.15]$} \\
\hline$\sigma_{R}$, monetary policy & 0.22 & 0.02 & {$[0.19,0.25]$} \\
\hline$\sigma_{\tau}, \operatorname{tax}$ & 0.7 & 0.07 & {$[0.59,0.81]$} \\
\hline$\sigma_{\pi}$, inflation target & 0.09 & 0.05 & {$[0.3,0.15]$} \\
\hline$\sigma_{b}$, debt/output target & 0.65 & 0.49 & {$[0.17,1.44]$} \\
\hline \multicolumn{4}{|l|}{ Steady state } \\
\hline$a:=100(\bar{a}-1)$, technology & 0.43 & 0.08 & {$[0.31,0.56]$} \\
\hline$\pi:=100(\bar{\pi}-1)$, inflation & 1.1 & 0.1 & {$[0.94,1.26]$} \\
\hline
\end{tabular}


Table 6: Posterior distributions for estimated parameters (Regime F) - continued

\begin{tabular}{llll}
\hline \hline & \multicolumn{2}{c}{ Posterior } \\
\cline { 2 - 4 } Parameter & Mean & SD & 90 percent credible set \\
\hline$b:=100 b$, debt/output & 36.63 & 2.01 & {$[33.33,39.93]$} \\
$\tau:=100 \bar{\tau}$, tax/output & 24.92 & 0.42 & {$[24.26,25.6]$} \\
$g:=100 \bar{g}$, govt spending/output & 24.4 & 0.4 & {$[23.78,25.05]$}
\end{tabular}

Note: Means, and standard deviations are over 50 independent runs of the SMC algorithm with $N=14,000, N_{\delta}=500, \lambda=2.5, N_{\text {blocks }}=6$, and $M_{M H}=1$. We compute $90 \%$ highest posterior density intervals. 

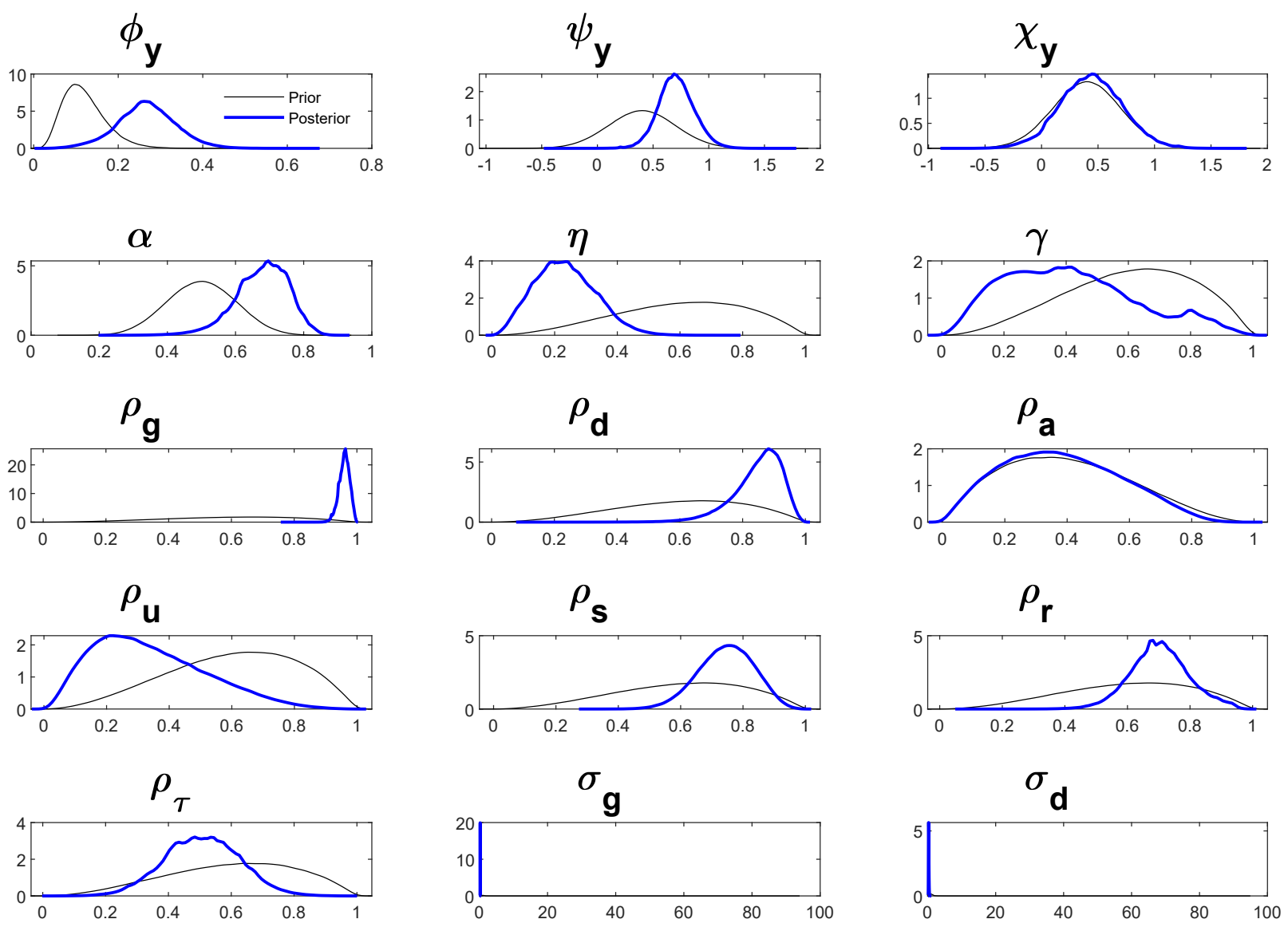

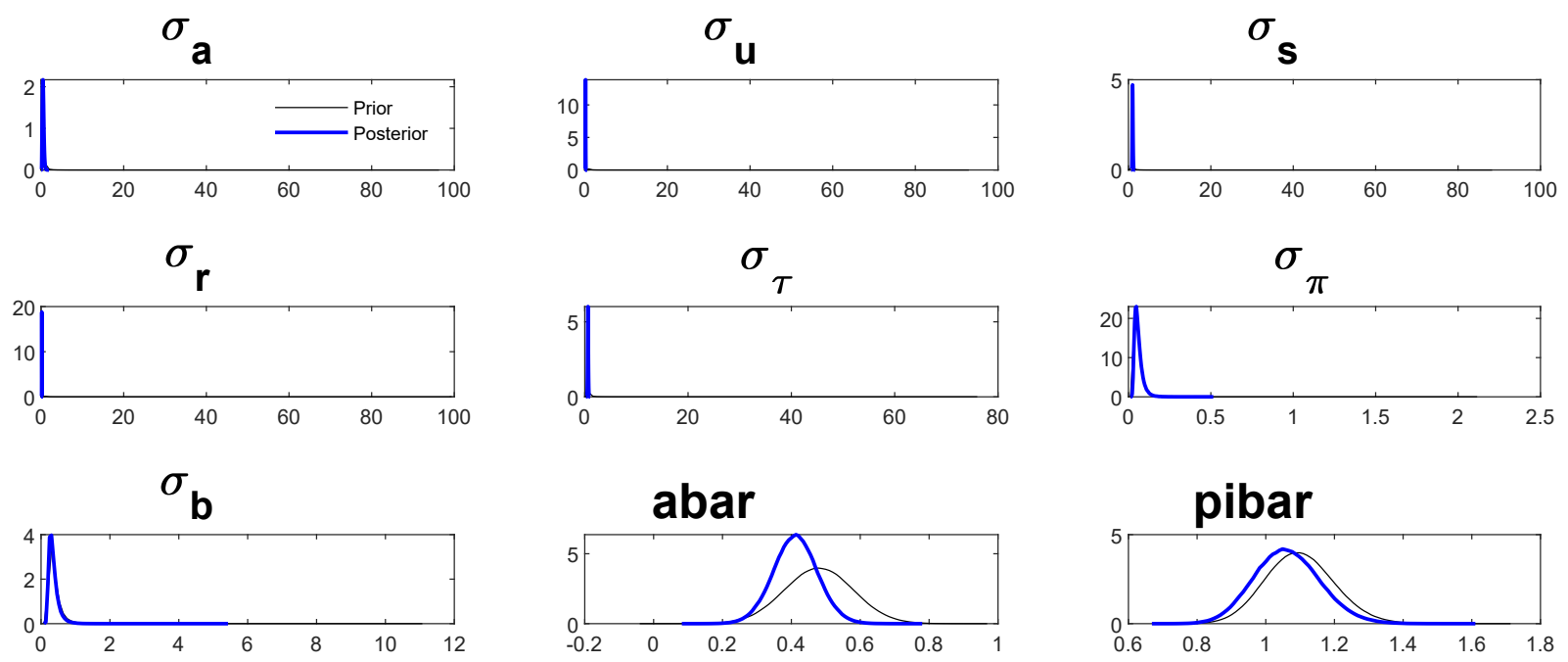

\section{mubar}
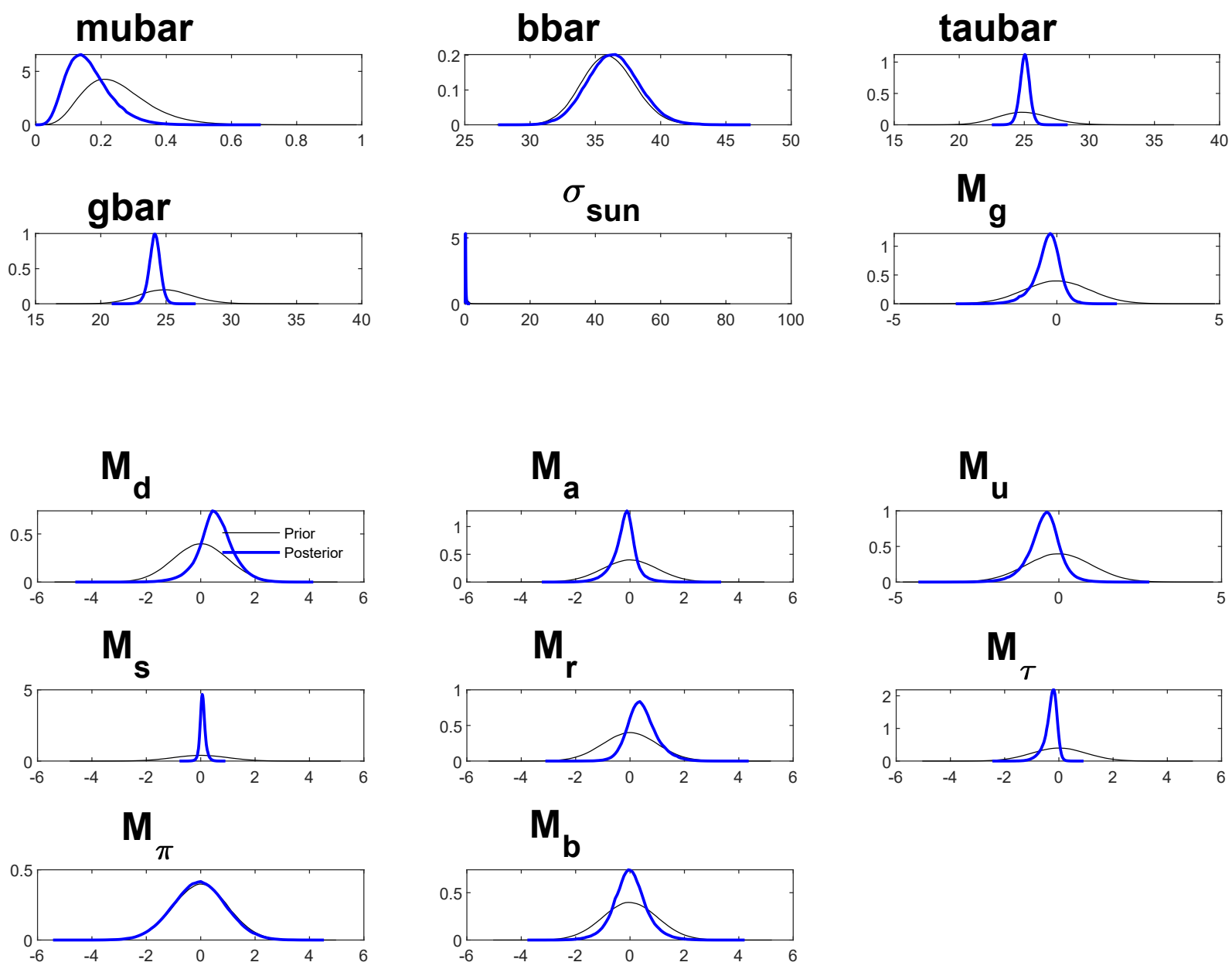

Figure 14: Prior and posterior densities of the estimated model parameters for the indeterminacy regime. The blue bold line depicts the posterior density, the black line the prior density. The prior densities are specified as in Bhattarai et al. (2016). 
Table 7: Posterior distributions for estimated parameters (Indeterminacy)

\begin{tabular}{|c|c|c|c|}
\hline \multirow{2}{*}{ Parameter } & \multicolumn{3}{|r|}{ Posterior } \\
\hline & Mean & SD & 90 percent credible set \\
\hline \multicolumn{4}{|l|}{ Monetary policy } \\
\hline$\phi_{\pi}$, interest rate response to inflation & 0.31 & 0.15 & {$[0.06,0.56]$} \\
\hline$\phi_{\pi}^{*}$, distance to monetary boundary & 0.71 & 0.05 & {$[0.66,0.79]$} \\
\hline$\phi_{Y}$, interest rate response to output & 0.28 & 0.02 & {$[0.25,0.31]$} \\
\hline$\rho_{R}$, response to lagged interest rate & 0.7 & 0.03 & {$[0.66,0.74]$} \\
\hline \multicolumn{4}{|l|}{ Fiscal policy } \\
\hline$\psi_{b}$, tax response to lagged debt & 0.05 & 0.02 & {$[0.008,0.08]$} \\
\hline$\psi_{b}^{*}$, distance to fiscal boundary & 0.05 & 0.01 & {$[0.039,0.055]$} \\
\hline$\psi_{Y}$, tax response to output & 0.71 & 0.03 & {$[0.66,0.77]$} \\
\hline $\begin{array}{l}\chi_{Y}, \text { govt spending response to } \\
\text { lagged output }\end{array}$ & 0.44 & 0.07 & {$[0.33,0.54]$} \\
\hline$\rho_{g}$, response to lagged govt spending & 0.96 & 0.004 & {$[0.957,0.967]$} \\
\hline$\rho_{\tau}$, response to lagged taxes & 0.5 & 0.03 & {$[0.44,0.54]$} \\
\hline \multicolumn{4}{|l|}{ Preference and $\mathrm{HHs}$} \\
\hline$\eta$, habit formation & 0.23 & 0.02 & {$[0.21,0.28]$} \\
\hline$\mu:=100\left(\beta^{-1}-1\right)$, discount factor & 0.16 & 0.01 & {$[0.14,0.18]$} \\
\hline \multicolumn{4}{|l|}{ Frictions } \\
\hline$\alpha$, price stickiness & 0.68 & 0.02 & {$[0.65,0.72]$} \\
\hline$\gamma$, price indexation & 0.4 & 0.08 & {$[0.3,0.49]$} \\
\hline \multicolumn{4}{|l|}{ Shocks } \\
\hline$\rho_{d}$, preference & 0.85 & 0.02 & {$[0.82,0.88]$} \\
\hline$\rho_{a}$, technology & 0.37 & 0.06 & {$[0.27,0.44]$} \\
\hline$\rho_{u}$, cost-push & 0.33 & 0.05 & {$[0.27,0.41]$} \\
\hline$\rho_{s}$, transfers & 0.75 & 0.02 & {$[0.73,0.77]$} \\
\hline$\sigma_{g}$, govt spending & 0.23 & 0.002 & {$[0.226,0.23]$} \\
\hline$\sigma_{d}$, preference & 0.29 & 0.02 & {$[0.26,0.32]$} \\
\hline$\sigma_{a}$, technology & 0.52 & 0.07 & {$[0.42,0.61]$} \\
\hline$\sigma_{u}$, cost-push & 0.21 & 0.006 & {$[0.2,0.21]$} \\
\hline$\sigma_{s}$, transfers & 1.02 & 0.008 & {$[1,1.03]$} \\
\hline$\sigma_{R}$, monetary policy & 0.18 & 0.006 & {$[0.17,0.19]$} \\
\hline$\sigma_{\tau}, \operatorname{tax}$ & 0.62 & 0.01 & {$[0.6,0.64]$} \\
\hline$\sigma_{\pi}$, inflation target & 0.06 & 0.004 & {$[0.05,0.06]$} \\
\hline$\sigma_{b}$, debt/output target & 0.36 & 0.02 & {$[0.32,0.39]$} \\
\hline \multicolumn{4}{|l|}{ Steady state } \\
\hline$a:=100(\bar{a}-1)$, technology & 0.41 & 0.01 & {$[0.39,0.42]$} \\
\hline$\pi:=100(\bar{\pi}-1)$, inflation & 1.06 & 0.02 & {$[1.03,1.07]$} \\
\hline
\end{tabular}


Table 7: Posterior distributions for estimated parameters (Indeterminacy) - continued

\begin{tabular}{llll}
\hline \hline & & \multicolumn{2}{c}{ Posterior } \\
\cline { 2 - 4 } Parameter & Mean & SD & 90 percent credible set \\
\hline$b:=100 b$, debt/output & 36.4 & 0.31 & {$[35.97,36.77]$} \\
$\tau:=100 \bar{\tau}$, tax/output & 25.06 & 0.09 & {$[24.94,25.17]$} \\
$g:=100 \bar{g}$, govt spending/output & 24.13 & 0.08 & {$[24.04,24.28]$} \\
& & & \\
Indeterminacy & & & \\
$\sigma_{\zeta}$, sunspot shock & 0.26 & 0.05 & {$[0.22,0.3]$} \\
$M_{g \zeta}$ & -0.29 & 0.11 & {$[-0.43,-0.13]$} \\
$M_{d \zeta}$ & 0.6 & 0.2 & {$[0.42,0.92]$} \\
$M_{a \zeta}$ & -0.2 & 0.08 & {$[-0.34,-0.1]$} \\
$M_{u \zeta}$ & -0.44 & 0.15 & {$[-0.59,-0.25]$} \\
$M_{s \zeta}$ & 0.08 & 0.03 & {$[0.03,0.12]$} \\
$M_{R \zeta}$ & 0.43 & 0.18 & {$[0.22,0.68]$} \\
$M_{\tau \zeta}$ & -0.3 & 0.1 & {$[-0.46,-0.2]$} \\
$M_{\pi \zeta}$ & -0.05 & 0.16 & {$[-0.28,0.26]$} \\
$M_{b \zeta}$ & -0.006 & 0.13 & {$[-0.18,0.12]$} \\
\hline
\end{tabular}

Note: Means, and standard deviations are over 50 independent runs of the SMC algorithm with $N=14,000, N_{\delta}=500, \lambda=2.5, N_{\text {blocks }}=6$, and $M_{M H}=1$. We compute $90 \%$ highest posterior density intervals. 


\section{Appendix F.1.1 Restricted estimation - prior as in Section 3.1 with renormal- ized policy parameters}

To ensure that we completely impose a particular policy regime during estimation, we again follow Bhattarai et al. (2016) and estimate the model with the reparameterized policy parameters $\phi_{\pi}^{*}$ and $\psi_{b}^{*}$. $\phi_{\pi}^{*}$ follows a Gamma distribution with a mean of 0.5 and a standard deviation of $0.2 . \quad \psi_{b}^{*}$ is also Gamma-distributed and has a mean of 0.05 and a standard deviation of 0.04. The prior densities of the remaining parameters are specified as in Section 3.1.
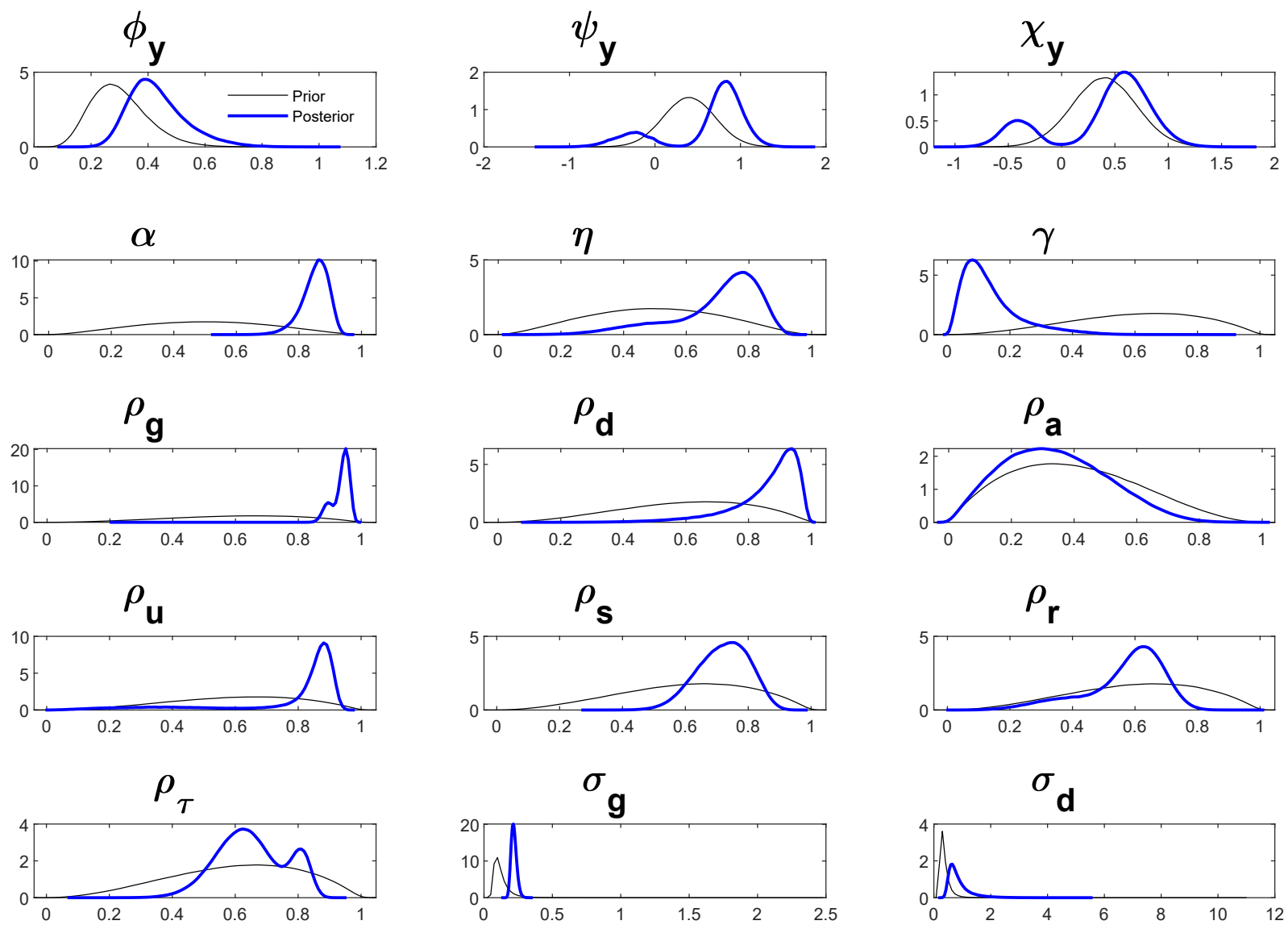

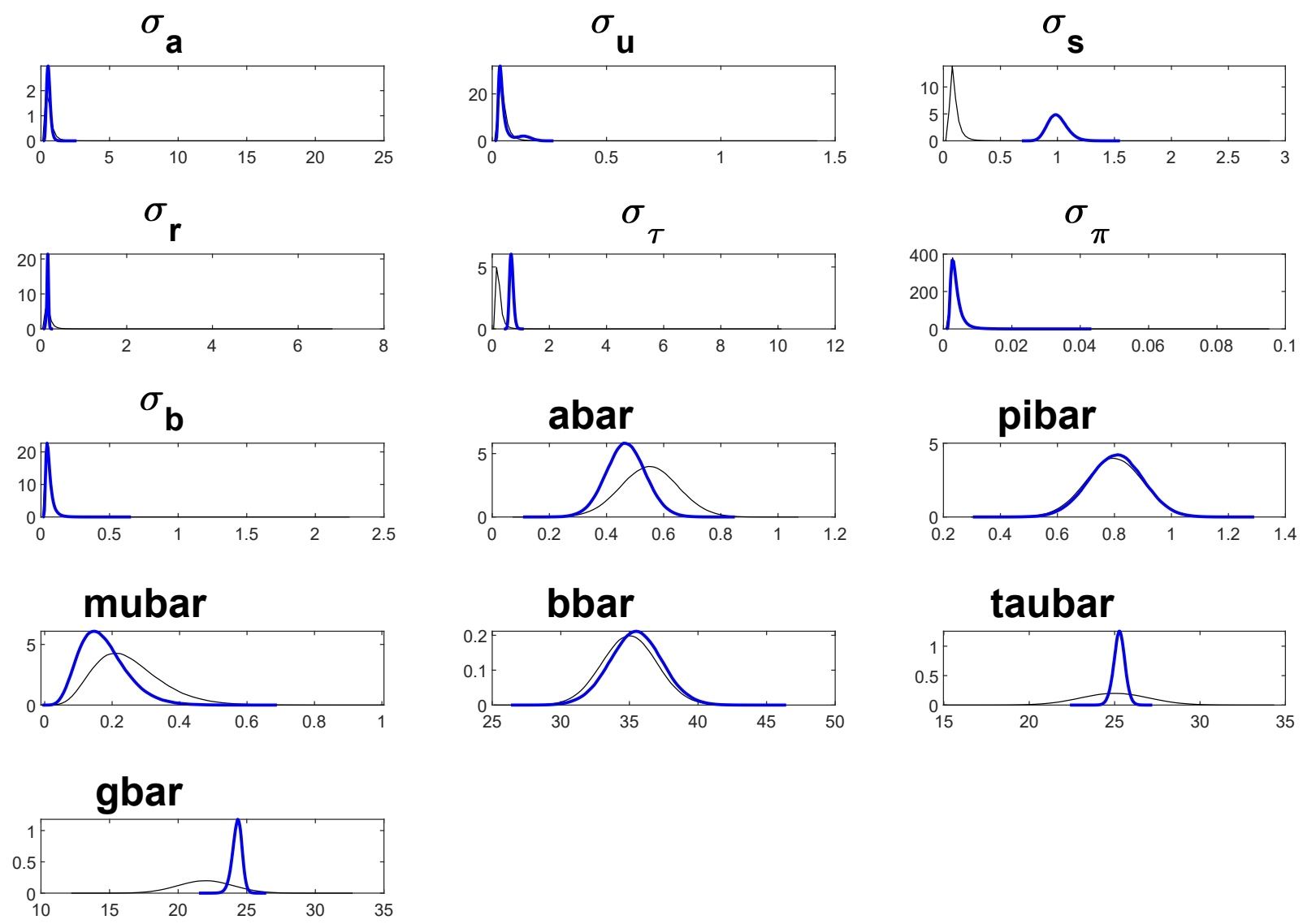

Figure 15: Prior and posterior densities of the estimated model parameters for regime F. The blue bold line depicts the posterior density, the black line the prior density. The densities of $\phi_{\pi}^{*}$ and $\psi_{b}^{*}$ are specified as in Bhattarai et al. (2016), the remaining parameters as in Section 3.1 .

Table 8: Posterior distributions for estimated parameters (Regime F)

\begin{tabular}{llll}
\hline \hline & \multicolumn{2}{c}{ Posterior } \\
\cline { 2 - 4 } Parameter & Mean & SD & 90 percent credible set \\
\hline Monetary policy & & & \\
$\phi_{\pi}$, interest rate response to inflation & 0.54 & 0.12 & {$[0.33,0.73]$} \\
$\phi_{\pi}^{*}$, distance to monetary boundary & 0.35 & 0.05 & {$[0.31,0.43]$} \\
$\phi_{Y}$, interest rate response to output & 0.44 & 0.06 & {$[0.4,0.54]$} \\
$\rho_{R}$, response to lagged interest rate & 0.56 & 0.09 & {$[0.38,0.63]$}
\end{tabular}

Fiscal policy

$\psi_{b}$, tax response to lagged debt

$\psi_{b}^{*}$, distance to fiscal boundary

$-0.02 \quad 0.01$

0.027

0.007

$[-0.04,-0.005]$

$\psi_{Y}$, tax response to output

0.58

0.39

$[0.02,0.04]$

$\chi_{Y}$, govt spending response to 0.38

0.36

$[-0.25,0.86]$

lagged output

$[-0.38,0.63]$ 
Table 8: Posterior distributions for estimated parameters (Regime F) - continued

\begin{tabular}{llll}
\hline \hline & \multicolumn{2}{c}{ Posterior } \\
\cline { 2 - 4 } Parameter & Mean & SD & 90 percent credible set \\
\hline$\rho_{g}$, response to lagged govt spending & 0.93 & 0.02 & {$[0.9,0.95]$} \\
$\rho_{\tau}$, response to lagged taxes & 0.66 & 0.07 & {$[0.61,0.79]$}
\end{tabular}

Preference and $\mathrm{HHs}$

$\eta$, habit formation

$\mu:=100\left(\beta^{-1}-1\right)$, discount factor

$\begin{array}{lll}0.69 & 0.1 & {[0.49,0.78]} \\ 0.17 & 0.01 & {[0.16,0.19]}\end{array}$

Frictions

$\alpha$, price stickiness

$\begin{array}{lll}0.85 & 0.02 & {[0.83,0.86]} \\ 0.13 & 0.06 & {[0.09,0.22]}\end{array}$

$\gamma$, price indexation

0.06

$[0.09,0.22]$

Shocks

$\begin{array}{llll}\rho_{d}, \text { preference } & 0.86 & 0.03 & {[0.82,0.9]} \\ \rho_{a}, \text { technology } & 0.33 & 0.04 & {[0.26,0.37]} \\ \rho_{u}, \text { cost-push } & 0.77 & 0.17 & {[0.45,0.88]} \\ \rho_{s}, \text { transfers } & 0.72 & 0.03 & {[0.65,0.74]} \\ \sigma_{g}, \text { govt spending } & 0.22 & 0.006 & {[0.21,0.23]} \\ \sigma_{d}, \text { preference } & 0.87 & 0.14 & {[0.58,1.03]} \\ \sigma_{a}, \text { technology } & 0.56 & 0.01 & {[0.55,0.58]} \\ \sigma_{u}, \text { cost-push } & 0.06 & 0.03 & {[0.04,0.12]} \\ \sigma_{s}, \text { transfers } & 1 & 0.003 & {[0.997,1.01]} \\ \sigma_{R}, \text { monetary policy } & 0.15 & 0.01 & {[0.13,0.16]} \\ \sigma_{\tau}, \text { tax } & 0.68 & 0.03 & {[0.66,0.72]} \\ \sigma_{\pi}, \text { inflation target } & 0.004 & 0 & {[0.0036,0.0039]} \\ \sigma_{b}, \text { debt/output target } & 0.06 & 0.001 & {[0.059,0.064]}\end{array}$

Steady state

\begin{tabular}{|c|c|c|c|}
\hline$a:=100(\bar{a}-1)$, technology & 0.47 & 0.007 & {$[0.46,0.48]$} \\
\hline$\pi:=100(\bar{\pi}-1)$, inflation & 0.81 & 0.02 & {$[0.79,0.83]$} \\
\hline$b:=100 \bar{b}$, debt/output & 35.5 & 0.16 & {$[35.28,35.62]$} \\
\hline$\tau:=100 \bar{\tau}$, tax/output & 25.26 & 0.12 & {$[25.05,25.36]$} \\
\hline$g:=100 \bar{g}$, govt spending/output & 24.31 & 0.09 & {$[24.24,24.45]$} \\
\hline
\end{tabular}

Note: Means, and standard deviations are over 50 independent runs of the SMC algorithm with $N=14,000, N_{\delta}=500, \lambda=2.5, N_{\text {blocks }}=6$, and $M_{M H}=1$. We compute $90 \%$ highest posterior density intervals. 

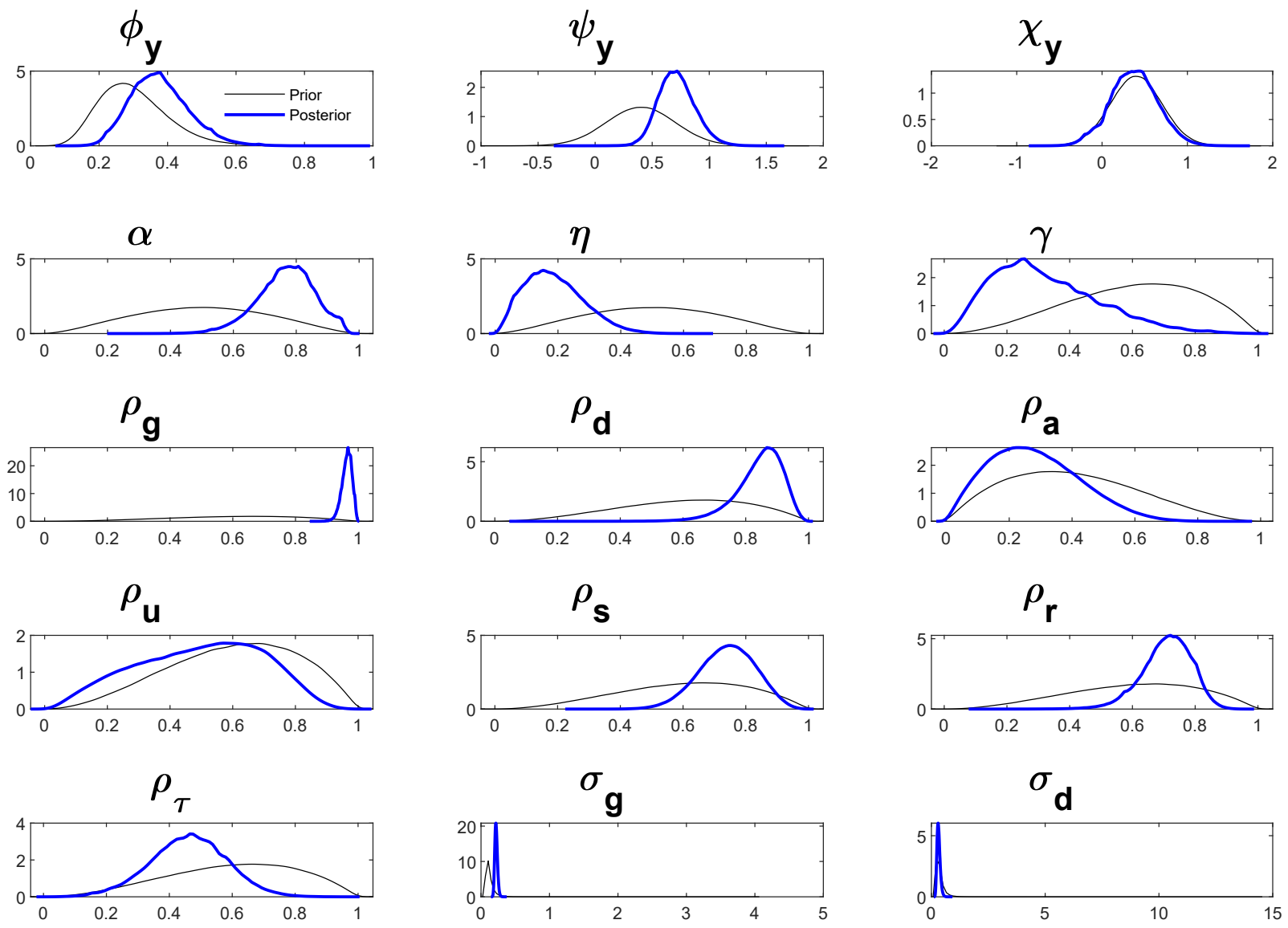

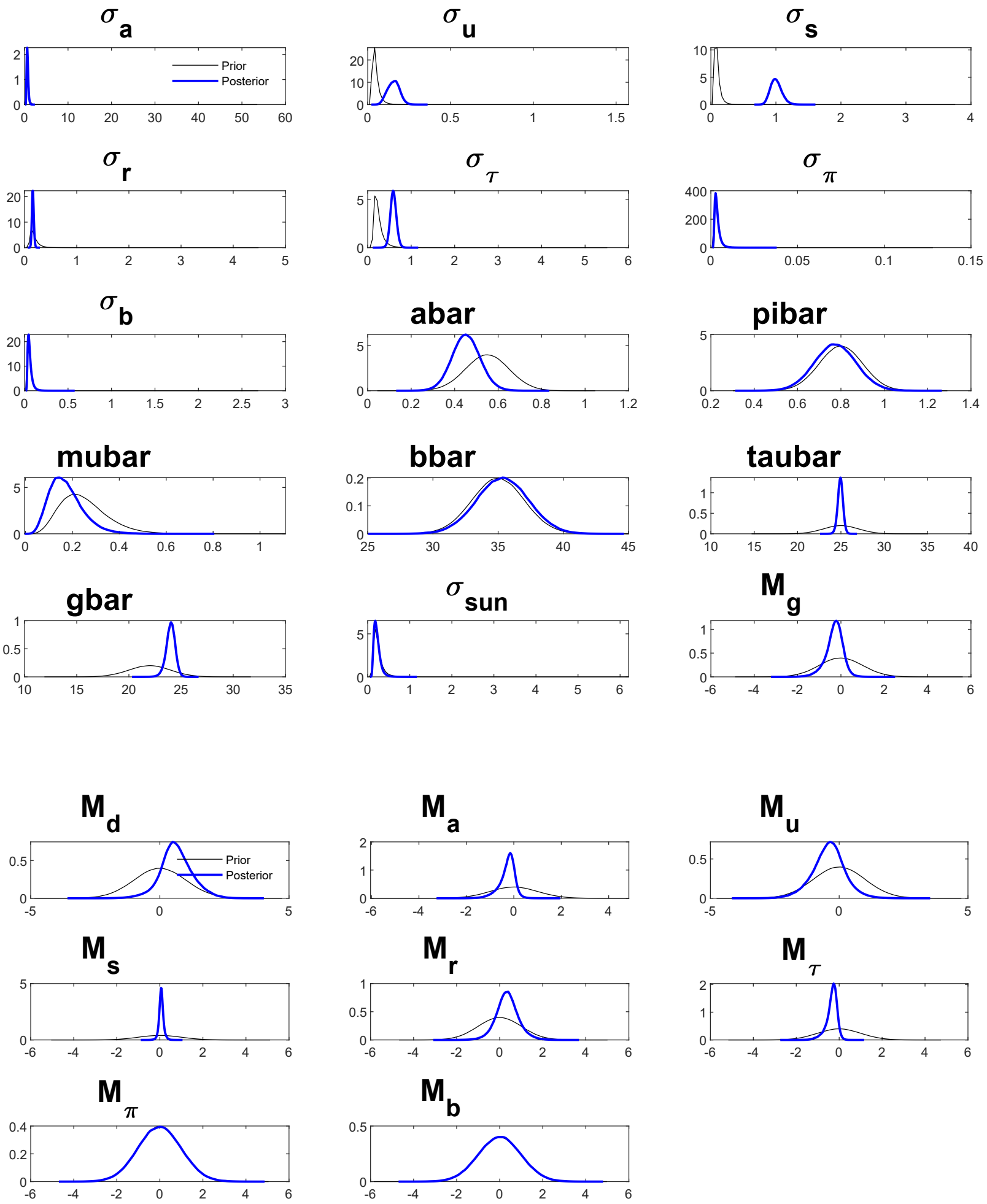

Figure 16: Prior and posterior densities of the estimated model parameters for the indeterminacy regime. The blue bold line depicts the posterior density, the black line the prior density. The densities of $\phi_{\pi}^{*}$ and $\psi_{b}^{*}$ are specified as in Bhattarai et al. (2016), the remaining parameters as in Section 3.1. 
Table 9: Posterior distributions for estimated parameters (Indeterminacy)

\begin{tabular}{|c|c|c|c|}
\hline \multirow[b]{2}{*}{ Parameter } & \multicolumn{3}{|r|}{ Posterior } \\
\hline & Mean & SD & 90 percent credible set \\
\hline \multicolumn{4}{|l|}{ Monetary policy } \\
\hline$\phi_{\pi}$, interest rate response to inflation & 0.11 & 0.19 & {$[-0.18,0.42]$} \\
\hline$\phi_{\pi}^{*}$, interest rate response to inflation & 0.86 & 0.05 & {$[0.83,0.95]$} \\
\hline$\phi_{Y}$, interest rate response to output & 0.39 & 0.02 & {$[0.36,0.41]$} \\
\hline$\rho_{R}$, response to lagged interest rate & 0.71 & 0.02 & {$[0.69,0.73]$} \\
\hline \multicolumn{4}{|l|}{ Fiscal policy } \\
\hline$\psi_{b}$, tax response to lagged debt & 0.05 & 0.02 & {$[0.02,0.09]$} \\
\hline$\psi_{b}^{*}$, distance to fiscal boundary & 0.06 & 0.004 & {$[0.05,0.06]$} \\
\hline$\psi_{Y}$, tax response to output & 0.73 & 0.03 & {$[0.7,0.78]$} \\
\hline $\begin{array}{l}\chi_{Y}, \text { govt spending response to } \\
\text { lagged output }\end{array}$ & 0.37 & 0.05 & {$[0.29,0.45]$} \\
\hline$\rho_{g}$, response to lagged govt spending & 0.97 & 0.002 & {$[0.962,0.969]$} \\
\hline$\rho_{\tau}$, response to lagged taxes & 0.45 & 0.03 & {$[0.4,0.49]$} \\
\hline \multicolumn{4}{|l|}{ Preference and $\mathrm{HHs}$} \\
\hline$\eta$, habit formation & 0.19 & 0.02 & {$[0.16,0.21]$} \\
\hline$\mu:=100\left(\beta^{-1}-1\right)$, discount factor & 0.17 & 0.01 & {$[0.16,0.19]$} \\
\hline \multicolumn{4}{|l|}{ Frictions } \\
\hline$\alpha$, price stickiness & 0.77 & 0.02 & {$[0.74,0.79]$} \\
\hline$\gamma$, price indexation & 0.31 & 0.04 & {$[0.22,0.35]$} \\
\hline \multicolumn{4}{|l|}{ Shocks } \\
\hline$\rho_{d}$, preference & 0.85 & 0.01 & {$[0.83,0.87]$} \\
\hline$\rho_{a}$, technology & 0.26 & 0.02 & {$[0.22,0.29]$} \\
\hline$\rho_{u}$, cost-push & 0.48 & 0.07 & {$[0.38,0.59]$} \\
\hline$\rho_{s}$, transfers & 0.74 & 0.01 & {$[0.73,0.76]$} \\
\hline$\sigma_{g}$, govt spending & 0.22 & 0.001 & {$[0.219,0.222]$} \\
\hline$\sigma_{d}$, preference & 0.31 & 0.01 & {$[0.29,0.33]$} \\
\hline$\sigma_{a}$, technology & 0.69 & 0.05 & {$[0.63,0.73]$} \\
\hline$\sigma_{u}$, cost-push & 0.16 & 0.01 & {$[0.15,0.18]$} \\
\hline$\sigma_{s}$, transfers & 1.01 & 0.006 & {$[0.99,1.01]$} \\
\hline$\sigma_{R}$, monetary policy & 0.16 & 0.003 & {$[0.155,0.163]$} \\
\hline$\sigma_{\tau}, \operatorname{tax}$ & 0.59 & 0.01 & {$[0.57,0.6]$} \\
\hline$\sigma_{\pi}$, inflation target & 0.004 & 0 & {$[0.003,0.004]$} \\
\hline$\sigma_{b}$, debt/output target & 0.06 & 0.004 & {$[0.056,0.068]$} \\
\hline \multicolumn{4}{|l|}{ Steady state } \\
\hline$a:=100(\bar{a}-1)$, technology & 0.45 & 0.008 & {$[0.44,0.46]$} \\
\hline$\pi:=100(\bar{\pi}-1)$, inflation & 0.77 & 0.01 & {$[0.75,0.79]$} \\
\hline
\end{tabular}


Table 9: Posterior distributions for estimated parameters (Indeterminacy) - continued

\begin{tabular}{llll}
\hline \hline & & \multicolumn{2}{c}{ Posterior } \\
\cline { 2 - 4 } Parameter & Mean & SD & 90 percent credible set \\
\hline$b:=100 b$, debt/output & 35.4 & 0.26 & {$[35.02,35.75]$} \\
$\tau:=100 \bar{\tau}$, tax/output & 24.01 & 0.06 & {$[24.82,24.99]$} \\
$g:=100 \bar{g}$, govt spending/output & 23.99 & 0.05 & {$[23.93,24.08]$} \\
& & & \\
Indeterminacy & & & \\
$\sigma_{\zeta}$, sunspot shock & 0.22 & 0.01 & {$[0.21,0.23]$} \\
$M_{g \zeta}$ & -0.28 & 0.06 & {$[-0.37,-0.2]$} \\
$M_{d \zeta}$ & 0.67 & 0.13 & {$[0.48,0.85]$} \\
$M_{a \zeta}$ & -0.26 & 0.07 & {$[-0.35,-0.19]$} \\
$M_{u \zeta}$ & -0.41 & 0.09 & {$[-0.54,-0.4]$} \\
$M_{s \zeta}$ & 0.07 & 0.02 & {$[0.04,0.09]$} \\
$M_{R \zeta}$ & 0.34 & 0.08 & {$[0.24,0.47]$} \\
$M_{\tau \zeta}$ & -0.35 & 0.08 & {$[-0.46,-0.25]$} \\
$M_{\pi \zeta}$ & -0.02 & 0.1 & {$[-0.18,0.15]$} \\
$M_{b \zeta}$ & 0 & 0.03 & {$[-0.11,0.14]$} \\
\hline
\end{tabular}

Note: Means, and standard deviations are over 50 independent runs of the SMC algorithm with $N=14,000, N_{\delta}=500, \lambda=2.5, N_{\text {blocks }}=6$, and $M_{M H}=1$. We compute $90 \%$ highest posterior density intervals. 


\section{Appendix F.2 Unrestricted estimation}

Here, we show plots of the prior and posterior densities for the remaining parameters from the unrestricted estimation with the SMC sampler and tables that summarize the estimation results. Here, the prior specification and the estimation approach corresponds to the
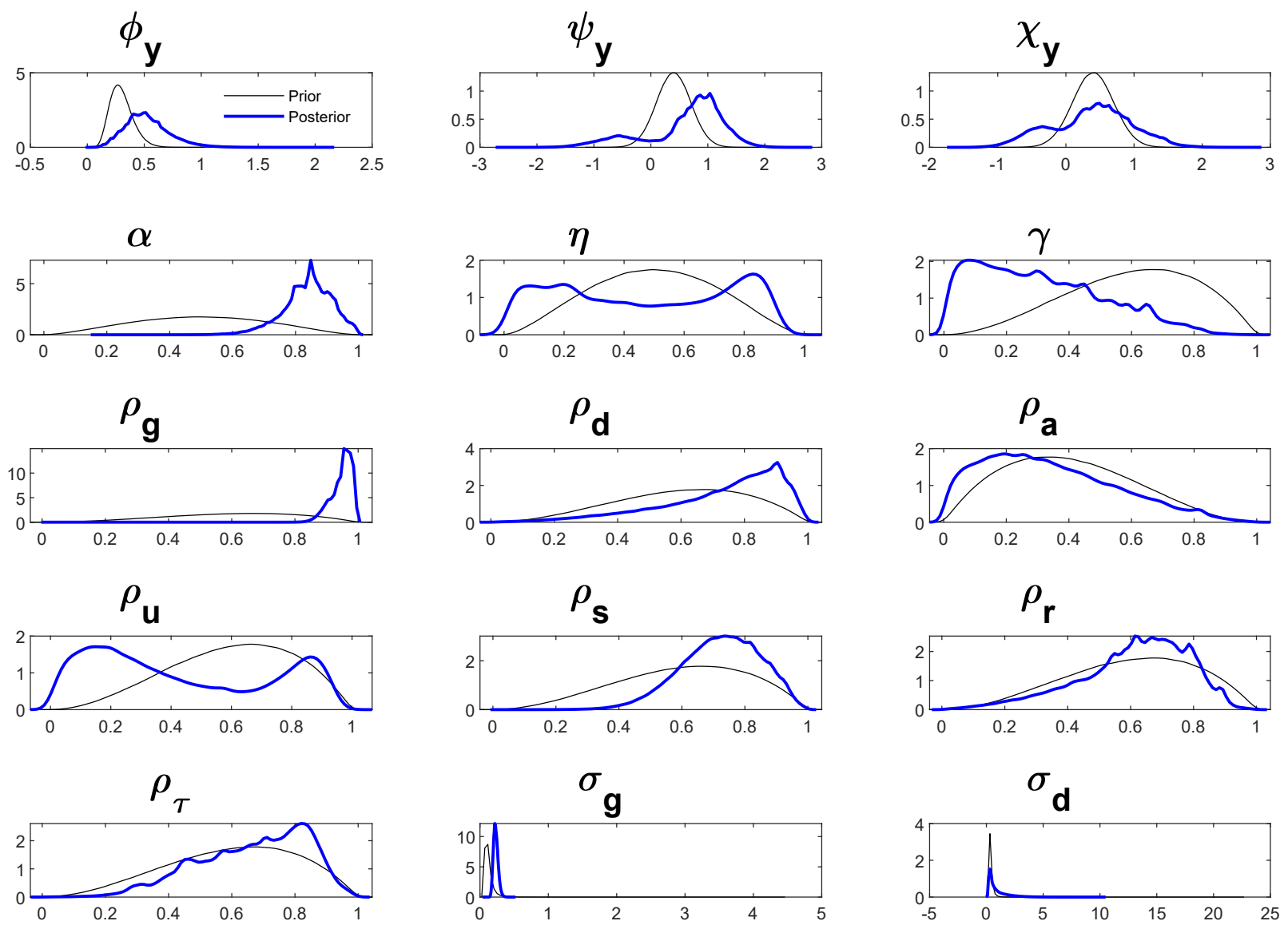
$\sigma_{\mathbf{a}}$
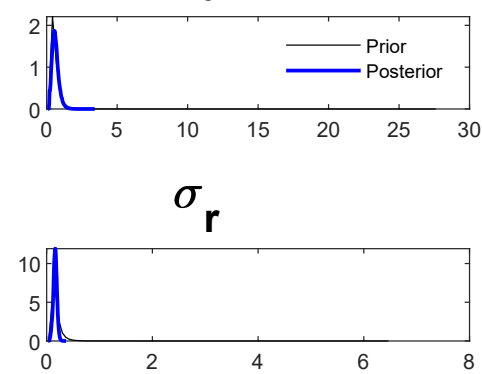

$\sigma_{\mathbf{b}}$

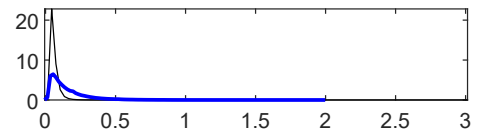

mubar

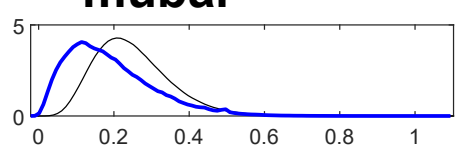

gbar

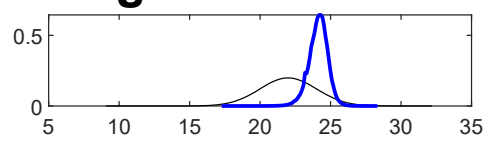

$\mathbf{M}_{\mathbf{d}}$

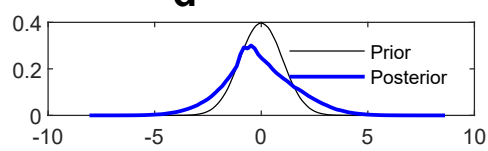

M

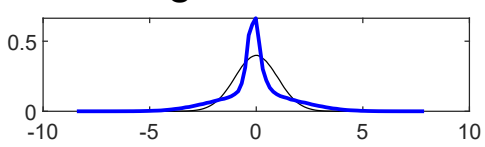

$\mathbf{M}$

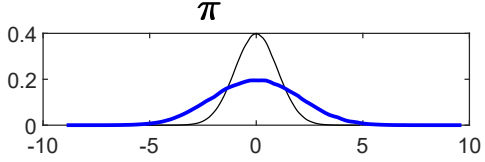

$\sigma_{\mathbf{u}}$

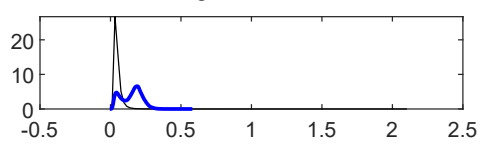

$\sigma_{\tau}$

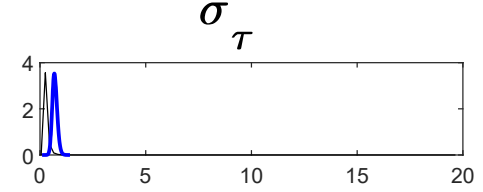

abar

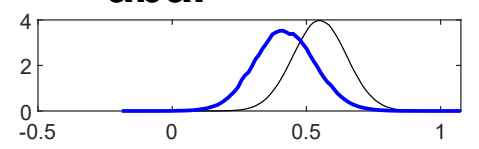

bbar

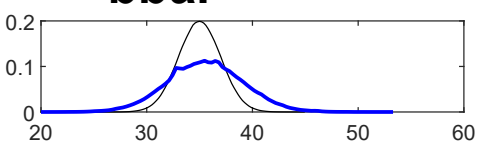

$\sigma_{\text {sun }}$

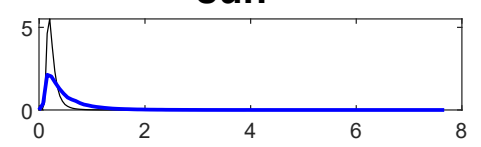

$\mathbf{M}_{\text {a }}$

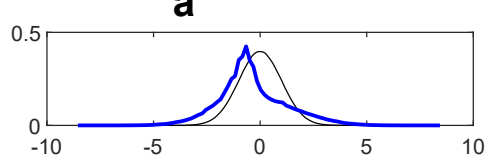

M

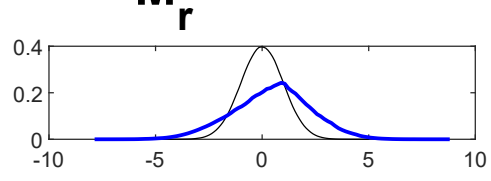

$\mathbf{M}_{\mathbf{b}}$

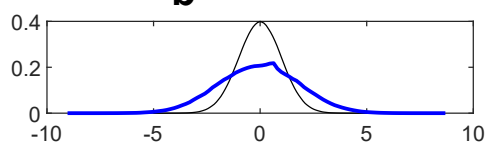

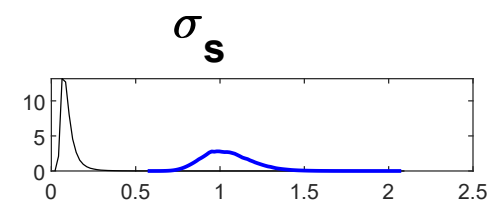
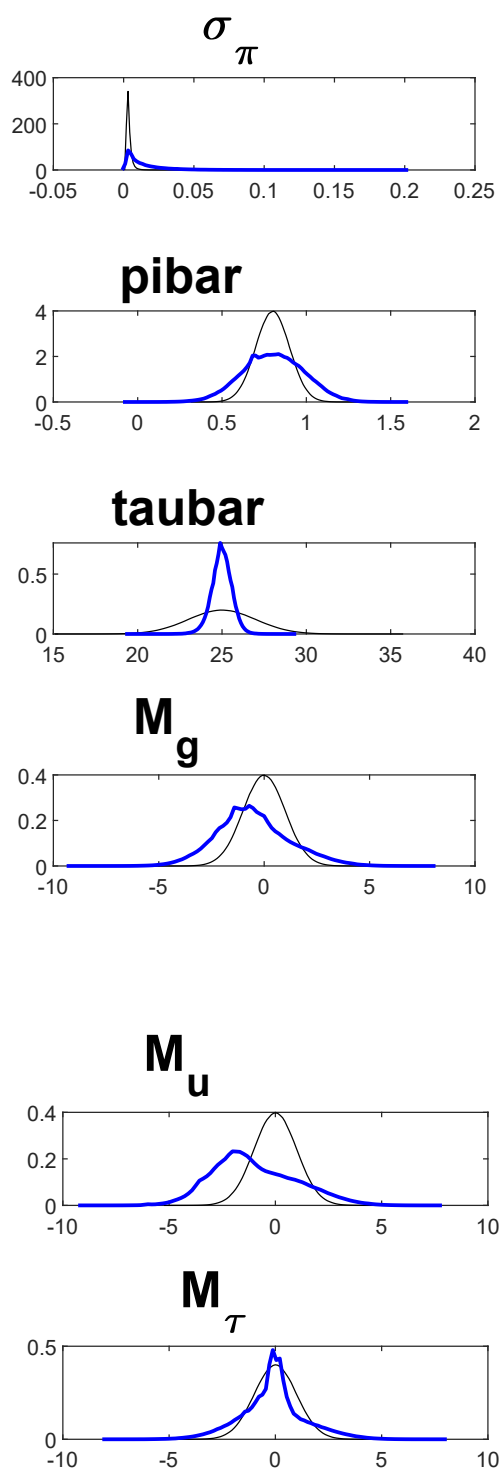

Figure 17: Prior and posterior densities of the estimated model parameters from the unrestricted estimation. The blue bold line depicts the posterior density, the black line the prior density. 
Table 10: Posterior distributions for estimated parameters (Unrestricted)

\begin{tabular}{llll}
\hline \hline & \multicolumn{2}{c}{ Posterior } \\
\cline { 2 - 4 } Parameter & Mean & SD & 90 percent credible set \\
\hline Monetary policy & & & \\
$\phi_{\pi}$, interest rate response to inflation & 0.4 & 0.22 & {$[0.13,0.73]$} \\
$\phi_{Y}$, interest rate response to output & 0.53 & 0.1 & {$[0.4,0.67]$} \\
$\rho_{R}$, response to lagged interest rate & 0.61 & 0.11 & {$[0.38,0.74]$}
\end{tabular}

Fiscal policy

$\psi_{b}$, tax response to lagged debt $\quad 0.026 \quad 0.04 \quad[-0.05,0.08]$

$\psi_{Y}$, tax response to output $\quad 0.62 \quad 0.5 \quad[-0.51,1.05]$

$\chi_{Y}$, govt spending response to $0.38 \quad 0.35 \quad[-0.25,0.86]$

lagged output

$\rho_{g}$, response to lagged govt spending $\quad 0.95 \quad 0.02 \quad[0.91,0.97]$

$\rho_{\tau}$, response to lagged taxes $\quad 0.66 \quad 0.11 \quad[0.5,0.81]$

Preference and $\mathrm{HHs}$

$\eta$, habit formation $\quad 0.45 \quad 0.23 \quad[0.20,0.81]$

$\mu:=100\left(\beta^{-1}-1\right)$, discount factor $\quad 0.19 \quad 0.04 \quad[0.14,0.22]$

Frictions

$\alpha$, price stickiness

$\begin{array}{lll}0.84 & 0.04 \quad[0.8,0.92]\end{array}$

$\gamma$, price indexation

$\begin{array}{lll}0.31 & 0.12 \quad[0.12,0.44]\end{array}$

Shocks

$\rho_{d}$, preference

$\begin{array}{lll}0.73 & 0.11 \quad[0.52,0.87]\end{array}$

$\rho_{a}$, technology

$\begin{array}{lll}0.33 & 0.08 & {[0.22,0.41]}\end{array}$

$\rho_{u}$, cost-push

$0.41 \quad 0.2$

$[0.15,0.71]$

$\rho_{s}$, transfers

0.72

0.04

$[0.64,0.77]$

$\sigma_{g}$, govt spending

$0.23 \quad 0.01$

$[0.22,0.24]$

$\sigma_{d}$, preference

$0.88 \quad 0.61$

$[0.31,1.78]$

$\sigma_{a}$, technology

0.62

0.09

$[0.52,0.72]$

$\sigma_{u}$, cost-push

0.15

0.05

$[0.09,0.22]$

$\sigma_{s}$, transfers

$1.04 \quad 0.02$

$[1,1.06]$

$\sigma_{R}$, monetary policy

0.16

0.02

$[0.13,0.18]$

$\sigma_{\tau}, \operatorname{tax}$

0.7

0.05

$[0.64,0.77]$

$\sigma_{\pi}$, inflation target

$0.006 \quad 0.006$

$[0.008,0.02]$

$\sigma_{b}$, debt/output target

0.15

0.05

$[0.11,0.2]$

Steady state

$a:=100(\bar{a}-1)$, technology

$0.42 \quad 0.03$

$[0.39,0.45]$

$\pi:=100(\bar{\pi}-1)$, inflation

0.8

0.05

$[0.74,0.87]$

$b:=100 \bar{b}$, debt/output

35.62

0.79

$[34.74,36.44]$

$\tau:=100 \bar{\tau}$, tax/output

24.97

0.18

[24.68, 25.2] 
Table 10: Posterior distributions for estimated parameters (Unrestricted) - continued

\begin{tabular}{llll}
\hline \hline \multirow{2}{*}{ Parameter } & \multicolumn{2}{c}{ Posterior } \\
\cline { 2 - 4 }$g:=100 \bar{g}$, govt spending/output & 24.12 & 0.21 & {$[23.82,24.48]$} \\
Indeterminacy & & & \\
$\sigma_{\zeta}$, sunspot shock & & & \\
$M_{g \zeta}$ & 0.49 & 0.14 & {$[0.27,0.68]$} \\
$M_{d \zeta}$ & -0.58 & 0.58 & {$[-1.43,0.03]$} \\
$M_{a \zeta}$ & -0.11 & 0.35 & {$[-0.69,0.33]$} \\
$M_{u \zeta}$ & -0.41 & 0.43 & {$[-0.94,0.17]$} \\
$M_{s \zeta}$ & -1.09 & 0.98 & {$[-2.37,0.03]$} \\
$M_{R \zeta}$ & -0.04 & 0.14 & {$[-0.28,0.16]$} \\
$M_{\tau \zeta}$ & 0.5 & 0.64 & {$[-0.21,1.22]$} \\
$M_{\pi \zeta}$ & -0.13 & 0.38 & {$[-0.7,0.22]$} \\
$M_{b \zeta}$ & 0 & 0.45 & {$[-0.54,0.46]$} \\
\hline Log Marginal data density & -0.07 & 0.29 & {$[-0.34,0.45]$} \\
\hline
\end{tabular}

Note: Means, standard deviations, and estimates of the log marginal data density are over 50 independent runs of the SMC algorithm with $N=20,000, N_{\delta}=600, \lambda=2.4$, $N_{\text {blocks }}=10$, and $M_{M H}=2$. We compute $90 \%$ highest posterior density intervals. The log marginal data density is obtained as a by-product during the correction step of the SMC algorithm, see Herbst and Schorfheide (2014) for further details. 